\title{
Planck early results. III. First assessment of the Low Frequency Instrument in-flight performance ${ }^{\star}$
}

A. Mennella ${ }^{22,37}$, M. Bersanelli22,39, R. C. Butler ${ }^{38}$, A. Curto ${ }^{47}$, F. Cuttaia ${ }^{38}$, R. J. Davis ${ }^{50}$, J. Dick ${ }^{57}$, M. Frailis ${ }^{37}$, S. Galeotta ${ }^{37}$, A. Gregorio ${ }^{23}$, H. Kurki-Suonio ${ }^{16,32}$, C. R. Lawrence ${ }^{49}$, S. Leach ${ }^{57}$, J. P. Leahy ${ }^{50}$, S. Lowe ${ }^{50}$, D. Maino ${ }^{22,39}$, N. Mandolesi ${ }^{38}$, M. Maris ${ }^{37}$, E. Martínez-González ${ }^{47}$, P. R. Meinhold ${ }^{19}$, G. Morgante ${ }^{38}$, D. Pearson ${ }^{49}$, F. Perrotta ${ }^{57}$, G. Polenta ${ }^{2,36}$, T. Poutanen ${ }^{32,16,1}$, M. Sandri ${ }^{38}$, M. D. Seiffert ${ }^{49,7}$, A.-S. Suur-Uski ${ }^{16,32}$, D. Tavagnacco ${ }^{37}$, L. Terenzi ${ }^{38}$, M. Tomasi ${ }^{22,39}$, J. Valiviita ${ }^{45}$, F. Villa ${ }^{38}$, R. Watson ${ }^{50}$, A. Wilkinson ${ }^{50}$, A. Zacchei ${ }^{37}$, A. Zonca ${ }^{19}$, B. Aja ${ }^{12}$, E. Artal $^{12}$, C. Baccigalupi ${ }^{57}$, A. J. Banday ${ }^{62,6,53}$, R. B. Barreiro ${ }^{47}$, J. G. Bartlett ${ }^{4,49}$, N. Bartolo ${ }^{20}$, P. Battaglia ${ }^{61}$, K. Bennett ${ }^{30}$, A. Bonaldi ${ }^{35}$, L. Bonavera ${ }^{57,5}$, J. Borrill ${ }^{52,59}$, F. R. Bouchet ${ }^{43}$, C. Burigana ${ }^{38}$, P. Cabella ${ }^{25}$, B. Cappellini ${ }^{39}$, X. Chen ${ }^{41}$, L. Colombo ${ }^{15,49}$, M. Cruz $^{13}$, L. Danese ${ }^{57}$, O. D’ Arcangelo ${ }^{48}$, R. D. Davies ${ }^{50}$, G. de Gasperis ${ }^{25}$, A. de Rosa ${ }^{38}$, G. de Zotti ${ }^{35,57}$, C. Dickinson ${ }^{50}$, J. M. Diego ${ }^{47}$, S. Donzelli ${ }^{39,45}$, G. Efstathiou ${ }^{44}$, T. A. Enßlin ${ }^{53}$, H. K. Eriksen ${ }^{45}$, M. C. Falvella ${ }^{3}$, F. Finelli ${ }^{38}$, S. Foley ${ }^{29}$, C. Franceschet ${ }^{22}$, E. Franceschi ${ }^{38}$, T. C. Gaier ${ }^{49}$, R. T. Génova-Santos ${ }^{46,27}$, D. George ${ }^{58}$, F. Gómez ${ }^{46}$, J. González-Nuevo ${ }^{57}$, K. M. Górski ${ }^{49,63}$, A. Gruppuso ${ }^{38}$, F. K. Hansen ${ }^{45}$, D. Herranz ${ }^{47}$, J. M. Herreros ${ }^{46}$, R. J. Hoyland ${ }^{46}$, N. Hughes ${ }^{9}$, J. Jewell ${ }^{49}$, P. Jukkala ${ }^{9}$, M. Juvela ${ }^{16}$, P. Kangaslahti ${ }^{49}$, E. Keihänen ${ }^{16}$, R. Keskitalo ${ }^{49,16}$, V.-H. Kilpia ${ }^{9}$, T. S. Kisner ${ }^{52}$, J. Knoche ${ }^{53}$, L. Knox ${ }^{18}$, M. Laaninen ${ }^{56}$, A. Lähteenmäki ${ }^{1,32}$, J.-M. Lamarre ${ }^{51}$, R. Leonardi2 ${ }^{28,30,19}$, J. León-Tavares ${ }^{1}$, P. Leutenegger ${ }^{61}$, P. B. Lilje ${ }^{45,8}$, M. López-Caniego ${ }^{47}$, P. M. Lubin ${ }^{19}$, M. Malaspina ${ }^{38}$, D. Marinucci ${ }^{26}$, M. Massardi ${ }^{35}$, S. Matarrese ${ }^{20}$, F. Matthai ${ }^{53}$, A. Melchiorri ${ }^{21}$, L. Mendes ${ }^{28}$, M. Miccolis ${ }^{61}$, M. Migliaccio ${ }^{25}$, S. Mitra ${ }^{49}$, A. Moss ${ }^{14}$, P. Natoli ${ }^{24,2,38}$, R. Nesti ${ }^{33}$, H. U. Nørgaard-Nielsen ${ }^{10}$, L. Pagano ${ }^{49}$, R. Paladini ${ }^{60,7}$, D. Paoletti ${ }^{38}$, B. Partridge ${ }^{31}$, F. Pasian ${ }^{37}$, V. Pettorino ${ }^{57}$, D. Pietrobon ${ }^{49}$, M. Pospieszalski ${ }^{55}$, G. Prézeau ${ }^{7,49}$, M. Prina ${ }^{49}$, P. Procopio ${ }^{38}$, J.-L. Puget ${ }^{42}$, C. Quercellini' ${ }^{25}$, J. P. Rachen ${ }^{53}$, R. Rebolo ${ }^{46,27}$, M. Reinecke ${ }^{53}$, S. Ricciardi ${ }^{38}$, G. Robbers ${ }^{53}$, G. Rocha ${ }^{49,7}$, N. Roddis ${ }^{50}$, J. A. Rubi no-Martín ${ }^{46,27}$, M. Savelainen ${ }^{16,32}$, D. Scott ${ }^{14}$, R. Silvestri ${ }^{61}$, A. Simonetto ${ }^{48}$, P. $\operatorname{Sjoman}^{9}$, G. F. Smoot ${ }^{17,52,4}$, C. Sozzi ${ }^{48}$, L. Stringhetti ${ }^{38}$, J. A. Tauber ${ }^{30}$, G. Tofani ${ }^{33}$, L. Toffolatti ${ }^{11}$, J. Tuovinen ${ }^{54}$, M. Türler ${ }^{40}$, G. Umana ${ }^{34}$, L. Valenziano ${ }^{38}$, J. Varis ${ }^{54}$, P. Vielva ${ }^{47}$, N. Vittorio ${ }^{25}$, L. A. Wade ${ }^{49}$, C. Watson ${ }^{29}$, S. D. M. White ${ }^{53}$, and F. Winder ${ }^{50}$

(Affiliations can be found after the references)

Received 9 January 2011 / Accepted 9 May 2011

\section{ABSTRACT}

The scientific performance of the Planck Low Frequency Instrument (LFI) after one year of in-orbit operation is presented. We describe the main optical parameters and discuss photometric calibration, white noise sensitivity, and noise properties. A preliminary evaluation of the impact of the main systematic effects is presented. For each of the performance parameters, we outline the methods used to obtain them from the flight data and provide a comparison with pre-launch ground assessments, which are essentially confirmed in flight.

Key words. cosmic background radiation - cosmology: observations - space vehicles: instruments - instrumentation: detectors

\section{Introduction}

The Planck ${ }^{1}$ mission was designed and developed to produce a full-sky survey of the cosmic microwave background (CMB)

\footnotetext{
* Corresponding author: A. Mennella, e-mail: aniello.mennella@fisica.unimi.it

1 Planck (http://www.esa.int/Planck) is a project of the European Space Agency (ESA) with instruments provided by two scientific consortia funded by ESA member states (in particular the lead countries France and Italy), with contributions from NASA (USA) and telescope reflectors provided by a collaboration between ESA and a scientific consortium led and funded by Denmark.
}

with unprecedented accuracy, both in temperature and polarisation. The need to separate astrophysical foregrounds required broad spectral coverage. Planck features nine frequency bands, roughly logarithmically spaced, in the range $27-900 \mathrm{GHz}$. The nine Planck sky maps will provide the community with a variety of new astrophysical data in this rich and largely unexplored frequency domain.

Two complementary cryogenic instruments employing different technologies, the Low Frequency Instrument (LFI) and the High frequency Instrument (HFI), share the Planck focal plane (Bersanelli et al. 2010; Mandolesi et al. 2010; Lamarre et al. 2010). The LFI is an array of coherent microwave receivers in 


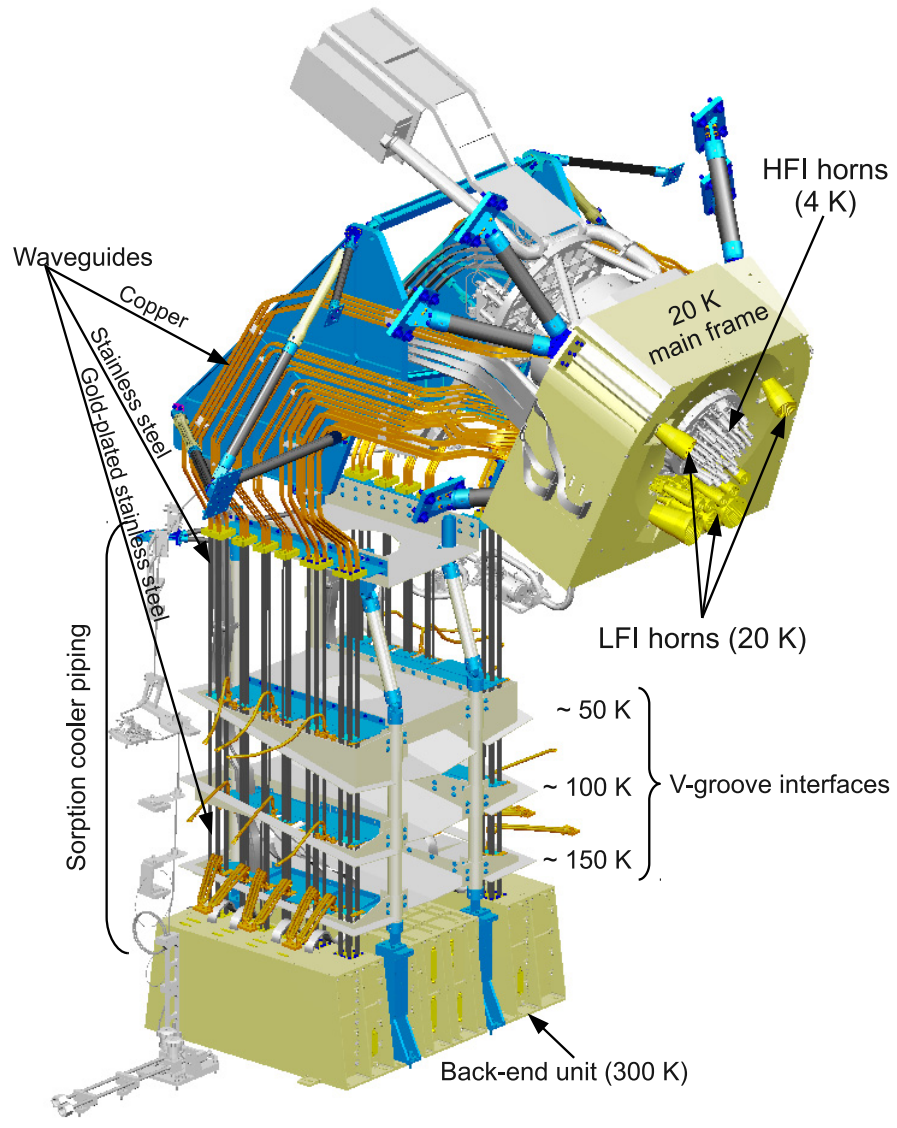

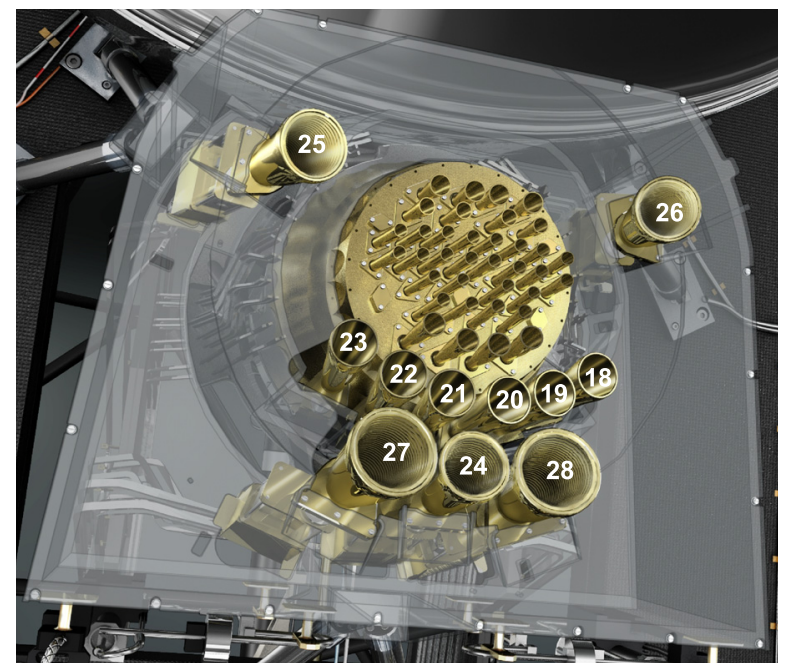

$70 \mathrm{GHz}$ : LFI-18 through LFI-23 $44 \mathrm{GHz}$ : LFI-24, LFI-25, LFI-26 $30 \mathrm{GHz}$ : LFI-27, LFI-28

Fig. 1. Left panel: 3D view of the LFI instrument with the main thermal stages, focal plane, waveguides, and sorption cooler piping highlighted. Right panel: labelling of feed horns (each feeding a RCA) on the LFI focal plane.

the $K \mathrm{a}, Q$, and $V$ frequency bands, based on state-of-the-art indium phosphide (InP) high electron mobility transistor (HEMT) amplifiers, implemented in a differential system using blackbody loads as reference signals. The LFI front-end is cooled to $\sim 20 \mathrm{~K}$ for optimal sensitivity, while the reference loads, connected with the HFI front-end unit, are cooled to $\sim 4.5 \mathrm{~K}$.

Following the successful launch of Planck on 14 May 2009, the LFI instrument has been working flawlessly and has been operated according to the mission plan (Planck Collaboration 2011a). Starting on 4 June 2009, the LFI entered the calibration and performance verification (CPV) phase, dedicated to instrument testing and radiometer tuning, exploiting the different thermal configuration during cool-down (Gregorio et al. 2011). Science observations began on 13 August 2009. Details of the Planck mission and scanning strategy are given in Planck Collaboration (2011a).

In this paper we describe the in-flight behaviour of the LFI and evaluate its performance during the first year of science operations. The LFI calibration strategy is based on a combination of ground and in-orbit measurements. Key instrument parameters have been measured both before launch and during flight, and can be compared for consistency. Ground calibration results have been obtained in three main test campaigns, corresponding to three levels of instrument integration, i.e., at single radiometer level (Villa et al. 2010), at instrument level (Mennella et al. 2010), and during the Planck system-level campaign carried out at $\mathrm{CSL}^{2}$. In the latter campaign (August 2008), the instruments were operated in conditions very similar to flight.

\footnotetext{
2 Centre Spatial de Liège.
}

Planck is based on a novel active cooling chain that supports for the first time space-borne $0.1 \mathrm{~K}$ bolometers and $20 \mathrm{~K}$ HEMT amplifiers. Much of the complexity of the Planck payload comes from its cryo-chain and from the very demanding stability requirements set by the two instruments (Planck Collaboration 2011b). The performance and stability of the LFI InP amplifiers and phase switches provide ground-breaking technological insight on these devices in view of possible future projects. The assessment of the instrument behaviour and systematics given here supports the Planck early release compact source catalog (ERCSC; Planck Collaboration 2011c) and the early science papers (see Planck papers in this volume). No analysis of polarisation performance is given here. A complete characterisation of the LFI instrument behaviour, including a full discussion of systematic effects and trend analysis, will be provided with the January 2013 data release.

\section{Instrument}

\subsection{Instrument configuration}

The instrument (Fig. 1) consists of a $\sim 20 \mathrm{~K}$ focal plane unit hosting the corrugated feed horns, orthomode transducers (OMTs), and receiver front-end modules (FEMs). A set of 44 composite waveguides (D'Arcangelo et al. 2009) interfaced with the three $V$-groove radiators (Planck Collaboration 2011b) connects the front-end modules to the warm $(\sim 300 \mathrm{~K})$ back-end unit (BEU), which contains further radio frequency amplification, 


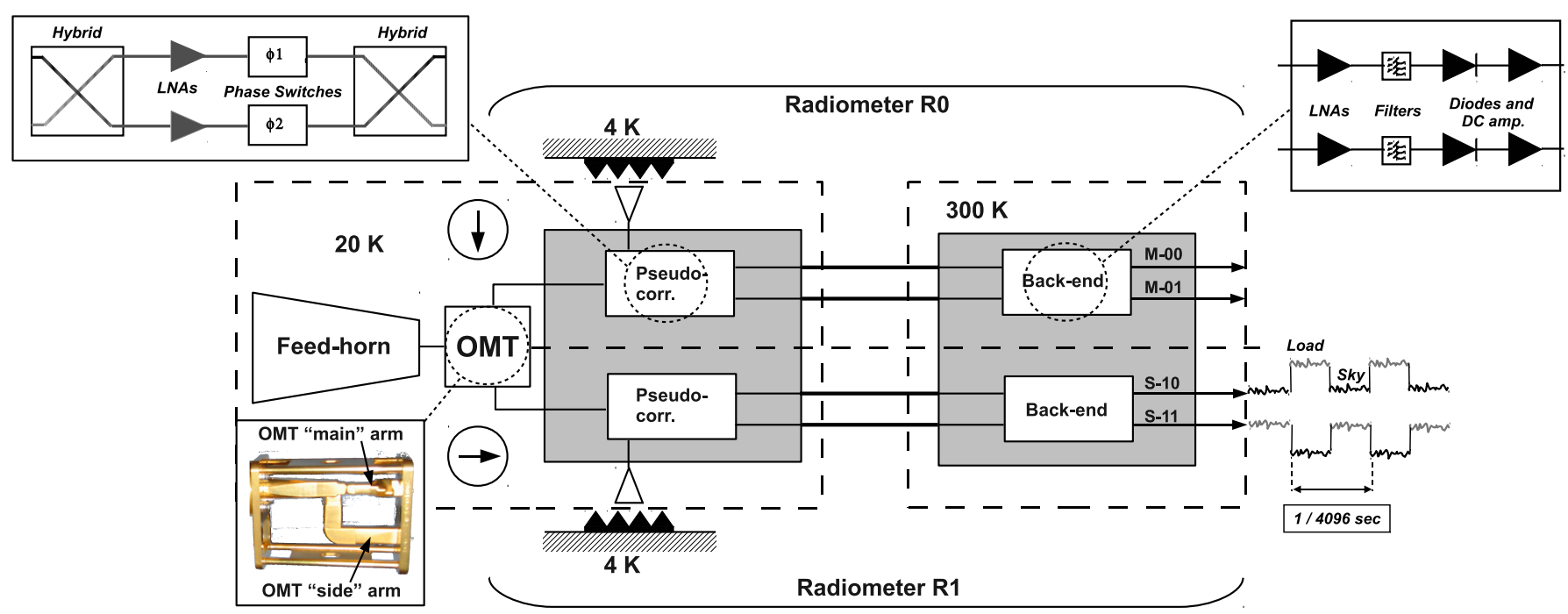

Fig. 2. Schematic of a complete RCA from feed-horn to analog voltage output, with insets showing the OMT, the details of the $20 \mathrm{~K}$ pseudocorrelator, and the details of the back-end radio-frequency amplification, low-pass filtering, detection, and DC amplification.

detector diodes, and electronics for data acquisition and bias supply. Each LFI radiometer chain assembly (RCA) consists of two radiometers, each feeding two diode detectors (Fig. 2), for a total of 44 detectors. The 11 RCAs are labelled by numbers from 18 to 28 as outlined in the right panel of Fig. 1.

Figure 2 shows a schematic of a complete RCA. The feed horn is connected to an OMT, which splits the incoming radiation into two perpendicular linear polarisation components that propagate through two independent pseudo-correlation differential radiometers. These radiometers are labelled as $\mathrm{M}$ or $\mathrm{S}$ depending on the arm of the OMT they are connected to ("Main" or "Side", see lower-left inset of Fig. 2).

In each radiometer, the sky signal coming from the OMT output is continuously compared with a stable $4 \mathrm{~K}$ blackbody reference load mounted on the external shield of the HFI $4 \mathrm{~K}$ box (Valenziano et al. 2009). After being summed by a first hybrid coupler, the two signals are amplified by $\sim 30 \mathrm{~dB}$. A phase shift alternating at $4096 \mathrm{~Hz}$ between $0^{\circ}$ and $180^{\circ}$ is applied in one of the two amplification chains. A second hybrid coupler separates back the sky and reference load components, which are further amplified and detected in the warm BEU. The output voltage ranges from $-2.5 \mathrm{~V}$ to $+2.5 \mathrm{~V}$.

The output diodes are labelled with binary codes $\Theta \otimes, Q 1$ (for radiometer $\mathrm{M}$ ) and 10,11 (for radiometer $\mathrm{S}$ ). The four outputs of each radiometric chain are referred to as $\mathrm{M}-0 \mathbf{0}, \mathrm{M}-\mathbf{0 1}, \mathrm{S}-10$, S-11 (Fig. 2).

After detection, an analog circuit in the data acquisition electronics (DAE) removes a programmable offset to obtain a nearly null DC output voltage, and a programmable gain is applied to match the signal level to the ADC input range.

After the ADC, data are downsampled, requantised, compressed according to a scheme described in Herreros et al. (2009) and Maris et al. (2009), and assembled into telemetry packets. On the ground, telemetry packets are converted to volts, using the applied offset and gain factors, and split into sky and reference load time-ordered data (TOD).

\subsection{Signal model}

In the ideal case of a perflectly balanced radiometer, the differential power output for each of the four diodes can be written

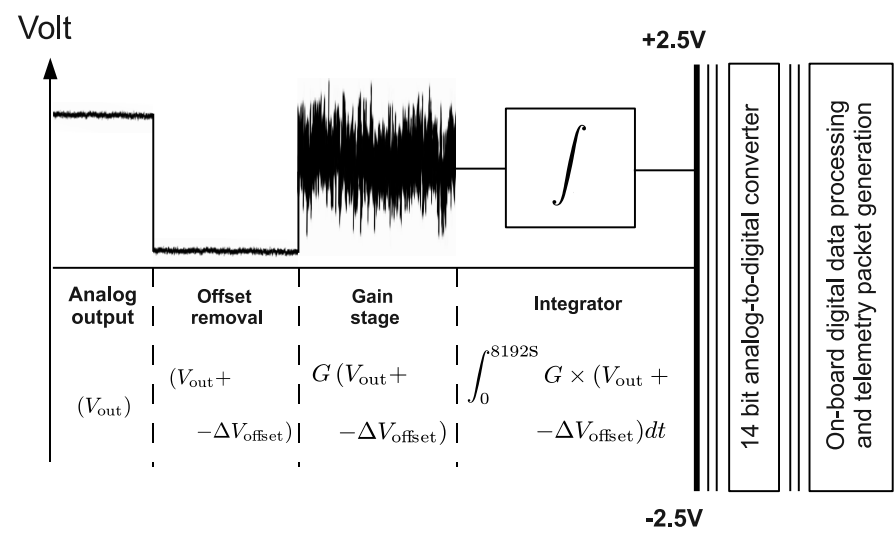

Fig. 3. Signal processing after detection with details about the ana$\log$ offset and gain stages. The analog signal is digitised by a 14 bit analog-to-digital converter (ADC) and then processed. Processing includes data rebinning, lossy requantisation, lossless compression, and telemetry packetization.

as follows (Seiffert et al. 2002; Mennella et al. 2003; Bersanelli et al. 2010):

$P_{\text {out }, 0}^{\text {diode }}=a G_{\text {tot }} k \beta\left[T_{\text {sky }}+T_{\text {noise }}-r\left(T_{\text {ref }}+T_{\text {noise }}\right)\right]$,

where $G_{\text {tot }}$ is the total gain, $k$ is the Boltzmann constant, $\beta$ is the bandwidth, and $a$ is the diode constant. $T_{\text {sky }}$ and $T_{\text {ref }}$ are the sky and reference load antenna temperatures at the inputs of the first hybrid, and $T_{\text {noise }}$ is the receiver noise temperature. In this section we always refer to average quantities. We omit, for simplicity, angled brackets, i.e., $T_{\text {sky }} \equiv\left\langle T_{\text {sky }}\right\rangle, T_{\text {ref }} \equiv\left\langle T_{\text {ref }}\right\rangle$, etc.

The gain modulation factor, $r$, is defined by

$r=\frac{T_{\text {sky }}+T_{\text {noise }}}{T_{\text {ref }}+T_{\text {noise }}}$

and is used to balance (in software) the temperature offset between the sky and reference load signals and minimise the residual $1 / f$ noise in the differential datastream. In nominal operating 
conditions, the gain modulation factor is in the range $0.84<r<$ 1.00 , depending on channel. This parameter is calculated each pointing period ${ }^{3}$ from the average uncalibrated total power data using the relationship:

$r=V_{\text {sky }} / V_{\text {ref }}$.

Details about the gain modulation factor calculation and its implementation in the scientific pipeline can be found in Mennella et al. (2003) and Zacchei et al. (2011).

The white noise spectral density at the output of each diode is essentially independent of the reference-load absolute temperature and is given by

$\Delta T_{0}^{\text {diode }}=\frac{2\left(T_{\text {sky }}+T_{\text {noise }}\right)}{\sqrt{\beta}}$.

If the front-end components are not perfectly balanced, then the separation of the sky and reference load signals after the second hybrid is not perfect and the outputs are mixed. First-order deviations in white noise sensitivity from the ideal behaviour are caused mainly by noise temperature and phase-switch amplitude mismatches. Following the notation used in Seiffert et al. (2002), we define $\epsilon_{T_{\mathrm{n}}}$, the imbalance in front end noise temperature, and $\epsilon_{A_{1}}$ and $\epsilon_{A_{2}}$, the imbalance in signal attenuation in the two states of the phase switch. Equation (4) for the two diodes of a slightly imbalanced radiometer then becomes

$\left(\Delta T^{\text {diode }}\right)^{2} \approx\left(\Delta T_{0}^{\text {diode }}\right)^{2}\left(1 \pm \frac{\epsilon_{A_{1}}-\epsilon_{A_{2}}}{2}+\alpha \epsilon_{T_{\mathrm{n}}}\right)$,

which is identical for the two diodes apart from the sign of the term $\left(\epsilon_{A_{1}}-\epsilon_{A_{2}}\right) / 2$, representing the phase switch amplitude imbalance. This indicates that the isolation loss caused by this imbalance generates an anticorrelation between the white noise levels of the single-diode data streams.

For this reason, the LFI scientific data streams are obtained by averaging the voltage outputs from the two diodes in each radiometer:

$V_{\mathrm{out}}^{\mathrm{rad}}=w_{1} V_{\mathrm{out}}^{\text {diode } 1}+w_{2} V_{\mathrm{out}}^{\text {diode } 2}$,

where $w_{1}$ and $w_{2}$ are inverse-variance weights calculated from the data as discussed in Zacchei et al. (2011). This way, the diode-diode anti-correlation is cancelled, and the radiometer white noise becomes

$\Delta T^{\mathrm{rad}} \approx \frac{\Delta T_{0}^{\text {diode }}}{\sqrt{2}}\left(1+\alpha \epsilon_{T_{\mathrm{n}}}\right)^{1 / 2}$.

In Eqs. (5) and (7), $\epsilon \ll 1$, while $\alpha$ is a term $\lesssim 1$ given by:

$\alpha=\frac{T_{\text {noise }}\left(2 T_{\text {noise }}+T_{\text {sky }}+T_{\text {ref }}\right)}{2\left(T_{\text {sky }}+T_{\text {noise }}\right)\left(T_{\text {ref }}+T_{\text {noise }}\right)}$.

Figure 4 illustrates this anti-correlated noise for a representative LFI channel (LFI28M). In black we plot the amplitude spectral density (ASD) of the weighted sum of the diode pair corresponding to the radiometer. The sky signal is visible as a series of spikes at the satellite rotation frequency and harmonics. The $1 / f$ and white noise levels are clearly visible, too. In red we show the ASD of the weighted difference of the same two diodes, a case in which (as expected) the sky signal is removed. In the difference signal the white noise is nearly $20 \%$ higher because of the anti-correlated noise in the output of two diodes. In fact, if the diodes were uncorrelated then the sum and difference of their ASD's should show identical white noise levels.

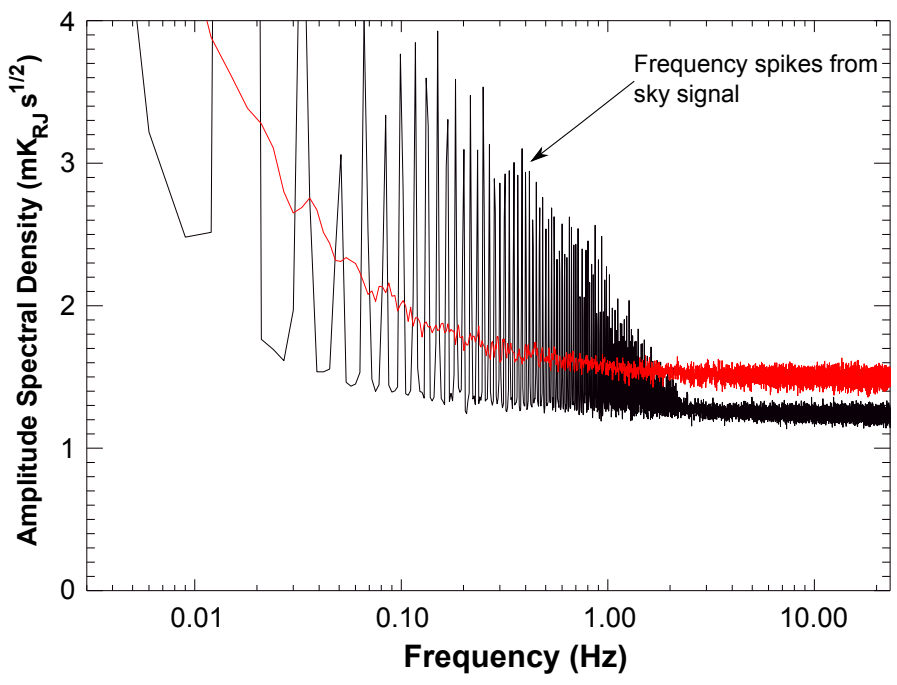

Fig. 4. Amplitude spectral density for weighted sum (black) and weighted difference (red) of LFI28M radiometer diodes. A white noise component that is anti-correlated between the two timestreams accounts for the difference in white noise level seen in the plot. Further analysis steps use the weighed sum data.

Table 1. LFI centre frequencies.

\begin{tabular}{|c|c|c|}
\hline $\mathrm{RCA}$ & $\begin{array}{c}\text { Radiometer M } \\
{[\mathrm{GHz}]}\end{array}$ & $\begin{array}{c}\text { Radiometer S } \\
{[\mathrm{GHz}]}\end{array}$ \\
\hline \multicolumn{3}{|l|}{$V$ band; "70 GHz" } \\
\hline $\begin{array}{r}\text { LFI18 } \ldots \ldots \ldots \\
\text { LFI19 } \ldots \ldots \ldots \\
\text { LFI20 } \ldots \ldots \ldots \\
\text { LFI } 21 \ldots \ldots \\
\text { LFI } 22 \ldots \ldots \\
\text { LFI } 23 \ldots \ldots \\
\text { Average } \ldots \ldots\end{array}$ & $\begin{array}{l}71.7 \\
67.5 \\
69.2 \\
70.4 \\
71.5 \\
70.8\end{array}$ & $\begin{array}{l}70.1 \\
69.6 \\
69.5 \\
69.5 \\
72.8 \\
71.3\end{array}$ \\
\hline \multicolumn{3}{|l|}{ Ka band; "44 GHz" } \\
\hline $\begin{array}{r}\text { LFI24 } \ldots \ldots \ldots \\
\text { LFI25 } \ldots \ldots \ldots \\
\text { LFI26 } \ldots \ldots \ldots \\
\text { Average } \ldots \ldots\end{array}$ & $\begin{array}{l}44.4 \\
44.0 \\
43.9\end{array}$ & $\begin{array}{l}44.1 \\
44.1 \\
44.1\end{array}$ \\
\hline \multicolumn{3}{|l|}{ K band; “30 GHz” } \\
\hline $\begin{array}{r}\text { LFI } 27 \ldots \ldots \ldots \\
\text { LFI2 } 28 \ldots \ldots \ldots \\
\text { Average } \ldots \ldots\end{array}$ & $\begin{array}{l}28.3 \\
28.8\end{array}$ & $\begin{array}{l}28.5 \\
28.2\end{array}$ \\
\hline
\end{tabular}

While the effects of this correlated component and the proper propagation through the pipeline to maps could be calculated and corrected at the map level, we have found it more natural and effective to combine the diodes in the time domain, performing calibration and further processing on the combined time stream.

\footnotetext{
${ }^{3}$ A pointing period is the amount of time during which the Planck telescope scans the same ring in the sky.
} 
A. Mennella et al.: Planck early results. III.

Table 2. Colour corrections for the 11 LFI RCAs individually and averaged by frequency.

\begin{tabular}{|c|c|c|c|c|c|c|c|}
\hline \multirow[b]{2}{*}{$\mathrm{RCA}$} & \multicolumn{7}{|c|}{ SPECTRAL INDEX $\alpha$} \\
\hline & -2.00 & -1.00 & 0.00 & 1.00 & 2.00 & 3.00 & 4.00 \\
\hline LFI18 & 1.054 & 1.028 & 1.011 & 1.003 & 1.003 & 1.010 & 1.026 \\
\hline LFI19 & 1.170 & 1.113 & 1.066 & 1.026 & 0.994 & 0.969 & 0.949 \\
\hline LFI20 & 1.122 & 1.079 & 1.044 & 1.017 & 0.997 & 0.983 & 0.975 \\
\hline LFI21 & 1.087 & 1.053 & 1.028 & 1.010 & 1.000 & 0.996 & 0.998 \\
\hline LFI22 & 0.973 & 0.971 & 0.976 & 0.988 & 1.007 & 1.033 & 1.066 \\
\hline LFI23 & 1.015 & 1.004 & 0.999 & 0.998 & 1.003 & 1.012 & 1.026 \\
\hline $70 \mathrm{GHz}$ average $\ldots \ldots$ & 1.070 & 1.041 & 1.021 & 1.007 & 1.001 & 1.001 & 1.007 \\
\hline LFI24 & 1.028 & 1.015 & 1.007 & 1.002 & 1.000 & 1.003 & 1.009 \\
\hline LFI25 & 1.039 & 1.024 & 1.013 & 1.005 & 1.000 & 0.999 & 1.000 \\
\hline LFI26 & 1.050 & 1.032 & 1.017 & 1.007 & 1.000 & 0.997 & 0.997 \\
\hline $44 \mathrm{GHz}$ average $\ldots \ldots$ & 1.039 & 1.024 & 1.012 & 1.004 & 1.000 & 0.999 & 1.002 \\
\hline LFI27 & 1.078 & 1.049 & 1.026 & 1.010 & 1.000 & 0.996 & 0.998 \\
\hline LFI28 & 1.079 & 1.049 & 1.026 & 1.009 & 1.000 & 0.997 & 1.002 \\
\hline $30 \mathrm{GHz}$ average & 1.079 & 1.049 & 1.026 & 1.010 & 1.000 & 0.997 & 1.000 \\
\hline
\end{tabular}

\subsection{In-band response and centre frequency}

The in-band receiver response has been thoroughly modelled and measured for all the LFI detectors during ground tests. The complete set of bandpass curves has been published in Zonca et al. (2009). From each curve we have derived the effective centre frequency according to

$v_{0}=\frac{\int_{v_{\min }}^{v_{\max }} v g(v) \mathrm{d} v}{\int_{v_{\min }}^{v_{\max }} g(v) \mathrm{d} v}$,

where $\Delta v=v_{\max }-v_{\min }$ is the receiver bandwidth and $g(v)$ is the bandpass response. Table 1 gives the centre frequencies of the 22 LFI radiometers. For each radiometer, $g(v)$ is calculated by weight-averaging the bandpass response of the two individual diodes with the same weights used to average detector timestreams. For simplicity and for historical reasons, we will continue to refer to the three channels as the 30,44 , and $70 \mathrm{GHz}$ channels.

Colour corrections, $C(\alpha)$, needed to derive the brightness temperature of a source with a power-law spectral index $\alpha$, are given in Table 2. The values are averaged for the 11 RCAs and for the three frequency channels. Details about the definition of colour corrections are provided in Zacchei et al. (2011).

\section{LFI operations}

\subsection{Cooldown and tuning}

LFI operations began during the calibration, performance, and verification (CPV) phase of the mission. Functionality and tuning tests (Gregorio et al. 2011) were carried out, taking advantage of the varying temperature of the $4 \mathrm{~K}$-stage during cooldown (see Fig. 5).

For optimal scientific performance, the bias voltages of the front-end low noise amplifiers (LNAs) and phase switch currents must be carefully tuned. Although radiometer tuning was performed during ground tests (Cuttaia et al. 2009), the procedure was repeated in flight to account for possible changes in the electrical and thermal environment.

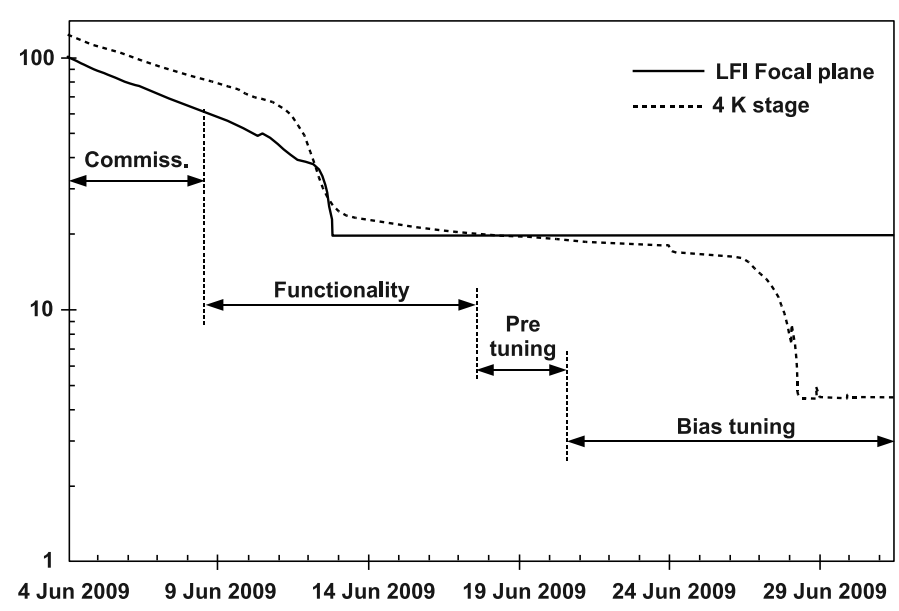

Fig. 5. Cooldown curve of LFI focal plane and $4 \mathrm{~K}$ stage. Functionality and bias tuning tests were carried out during this phase.

Phase switch bias currents $\left(I_{1}, I_{2}\right)$ of the $30 \mathrm{GHz}$ and $44 \mathrm{GHz}$ RCAs were tuned to optimise amplitude balance. This was done by exploring a two-dimensional bias surface around the optimal points found during ground tests. The results (repeated also at the end of the tuning campaign) confirmed the optimal points found before launch. The phase switches of the $70 \mathrm{GHz}$ RCAs, instead, were set to the maximum biases $(1 \mathrm{~mA})$ and were not tuned. Ground tests showed that the rise time was sensitive to bias currents, and decreased as the current increased from $0.5 \mathrm{~mA}$ to $1 \mathrm{~mA}$ (Gregorio et al. 2011).

LNA biases were tuned by exploring a large volume in the bias space of each amplifier. The common drain voltage, $V_{\mathrm{d}}$, the gate voltage of the first stage, $V_{\mathrm{g} 1}$, and the gate voltage common to the remaining stages, $V_{\mathrm{g} 2}$, were sampled according to a "hyper matrix" (HYM) tuning strategy, in which several bias quadruplets $\left[\left(V_{\mathrm{g} 1}, V_{\mathrm{g} 2}\right)_{\mathrm{LNA} 1},\left(V_{\mathrm{g} 1}, V_{\mathrm{g} 2}\right)_{\mathrm{LNA} 2}\right]$ were varied for each radiometer. This strategy increased considerably the sampled parameter space with respect to ground tuning tests (Cuttaia et al. 2009), and allowed us to fully characterise the radiometer 

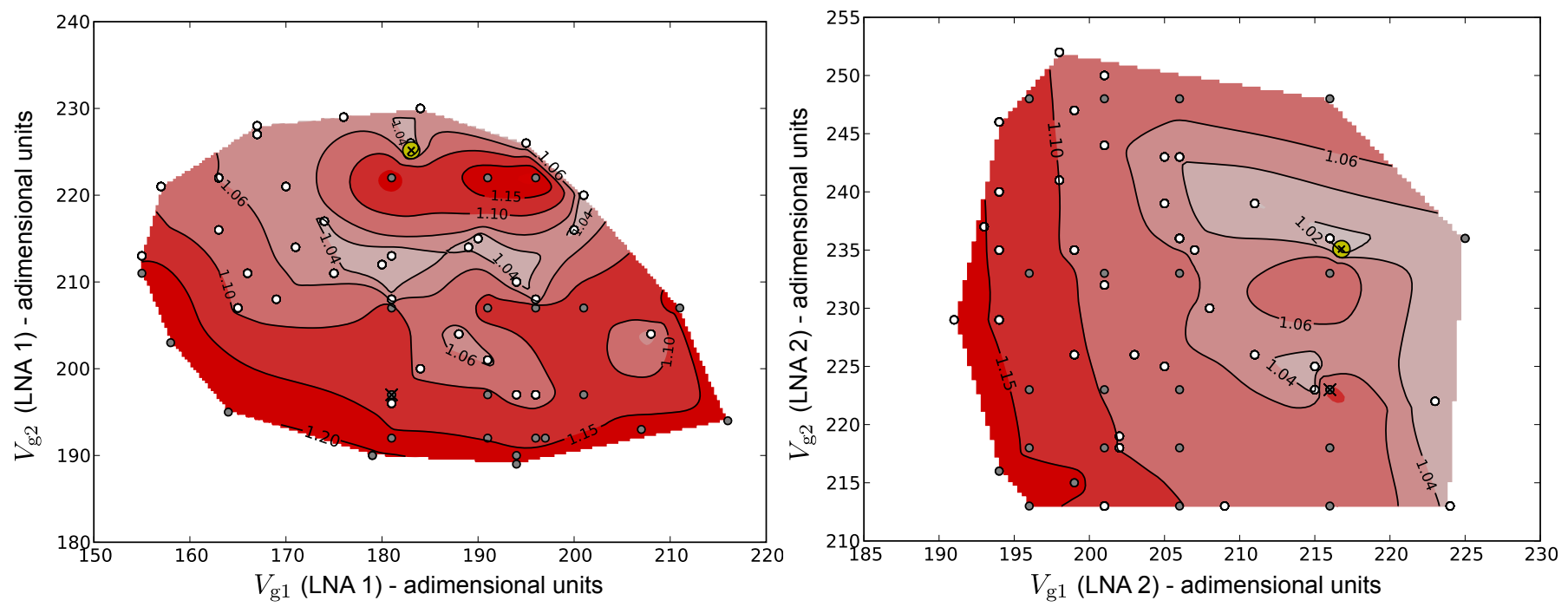

Fig. 6. Example of condensed noise temperature maps for the two LNAs of radiometer LFI21S. Contour values represent the ratio of the noise temperature in a given bias configuration to the minimum noise temperature found in all tested configurations. The yellow crossed point is the chosen bias point.

performance in terms of noise temperature, isolation, and drain current balance.

To define the broad bias regions to be deeply sampled by the HYM tuning procedure, a pre-tuning phase was run with the instrument at $20 \mathrm{~K}$ and the reference loads at $20.4 \mathrm{~K}$. The large imbalance between the sky and reference load signals provided us with enough voltage difference to estimate noise temperaure.

During the HYM tuning phase, smaller bias volumes around the optimal pre-tuning points were sampled at four different temperatures (see Fig. 5) between $19.1 \mathrm{~K}$ and $4 \mathrm{~K}$ during the cooldown of the ${ }^{4} \mathrm{He}-\mathrm{JT}$ cooler, allowing us also to characterize the response linearity (Mennella et al. 2009). Drain voltages were also tuned for a limited subset of $V_{\mathrm{g} 1}, V_{\mathrm{g} 2}$ quadruplets, making the overall bias space six-dimensional.

Figure 6 shows an example of "condensed noise temperature" maps. These are contour plots in the $V_{\mathrm{g} 1}, V_{\mathrm{g} 2}$ space for the LNAs of a given radiometer. Each point in the plot is the average of the best $20 \%$ noise temperature values determined by the quadruplets sharing that particular $V_{\mathrm{g} 1}, V_{\mathrm{g} 2}$ pair. The same approach was used to map isolation and drain currents.

Noise temperature and isolation maps were calculated by fitting data using both a linear and a non-linear response model. Minor effects caused by non-linear response were observed, as expected, only for 30 and $44 \mathrm{GHz}$ channels, confirming onground test results. As emphasized in Mennella et al. (2009), signal compression in 30 and $44 \mathrm{GHz}$ receivers is relevant only if the input range is of the order of $\gtrsim 1 \mathrm{~K}$. Therefore it can be completely neglected in normal operations and in flight calibration, where the sky dynamic range is $\lesssim 10 \mathrm{mK}$. The optimal bias values found during tuning were close to those found during satellitelevel tests on the ground, with maximum deviations of about $10 \%$. Table 3 summarizes the improvements in noise temperature and isolation between the ground and flight tests.

The only large change was in radiometer LFI21S, for which isolation improved from $-7 \mathrm{~dB}$ measured on ground to $-16 \mathrm{~dB}$ measured in flight. There was a corresponding improvement in white noise sensitivity (see Sect. 6.2).
Table 3. Reduction in $T_{\text {noise }}$ and increase in isolation (in $\mathrm{dB}$ ) in flight compared to ground tests.

\begin{tabular}{lccc}
\hline \hline & $\begin{array}{c}\text { Min } \\
{[\%]}\end{array}$ & $\begin{array}{c}\text { Median } \\
{[\%]}\end{array}$ & $\begin{array}{c}\operatorname{Max} \\
{[\%]}\end{array}$ \\
\hline$\delta T_{\text {noise }} / T_{\text {noise }} \ldots \ldots \ldots \ldots \ldots$ & 0 & 4.2 & 12.5 \\
$\delta$ isolation/isolation[dB] $\ldots \ldots \ldots$ & 1 & 6 & 115 \\
\hline
\end{tabular}

After tuning, an unexpectedly high level of $1 / f$ noise fluctuations was observed for the $44 \mathrm{GHz}$ RCAs, using either the new flight bias settings or the old ground ones. Dedicated tests showed that this instability was correlated with the phase switch configuration of $70 \mathrm{GHz}$ LFI23, and disappeared when the radiometers were biased with the optimal voltages found during the optimisation of the individual front-end modules before instrument integration (see Fig. 7). This interaction between RCAs belonging to different frequency channels was unexpected and deeply investigated during CPV. The root cause was never fully established, but the most likely explanation was a parasitic oscillation triggered by unexpected cross-talk in the warm electronics. Details about investigations performed in flight to understand and solve this problem are reported in Gregorio et al. (2011). The final bias setting (Davis et al. 2009), characterised by a slightly higher power consumption $(\sim 40 \mathrm{~mW})$ but similar noise and isolation performance, eliminated the problem.

Table 4 summarises the bias settings chosen for the frontend amplifiers at the end of CPV. They have never been changed since the start of nominal operations.

The last tuning step of the CPV phase configures the signal processing unit (SPU) data compressor for each of the 44 LFI channels (Maris et al. 2009) so that the data fit into the allocated telemetry bandwidth without significant loss of sensitivity (defined below) from quantisation errors produced by the lossy compressor. Quantisation errors appear as an additional white noise component that can be characterised as the ratio of the quantisation rms $\varepsilon_{\mathrm{q}}$ over the intrinsic rms $\sigma$ of the signal before 
A. Mennella et al.: Planck early results. III.

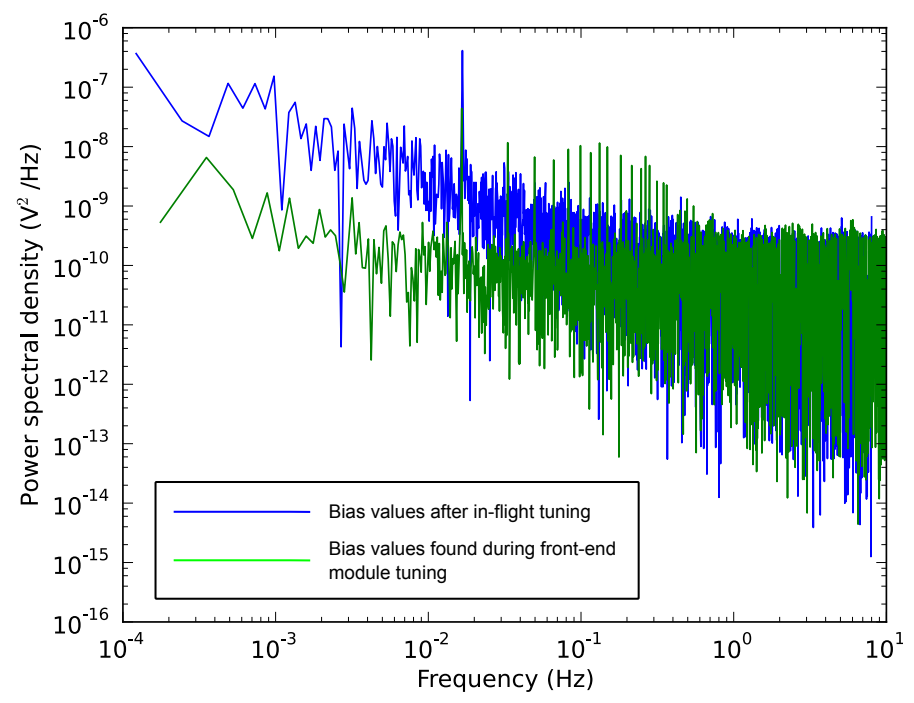

Fig. 7. Power spectral density of data from LFI25M-00 detector with two different bias sets (Blue: optimal biases determined after flight tuning tests. Green: optimal biases determined during front-end module tuning before integration).

Table 4. Origin of final bias settings.

\begin{tabular}{|c|c|c|}
\hline \multirow[b]{2}{*}{ RCA } & \multicolumn{2}{|c|}{ RADIOMETER } \\
\hline & M & $\mathrm{S}$ \\
\hline LFI18 . . . . & Flight $^{a}$ & Flight \\
\hline LFI19 . . . . . . . & Ground $^{b}$ & Ground \\
\hline LFI20 . . . . . . . & Ground & Ground \\
\hline LFI21 . . . . . . & Ground & Flight \\
\hline LFI22 & Ground & Ground \\
\hline LFI23 ... & Ground & Ground \\
\hline LFI24 . . & $\mathrm{FEM}^{c}$ & FEM \\
\hline LFI25 . . & FEM & FEM \\
\hline LFI26 . . . & FEM & FEM \\
\hline LFI27 . & Ground & Ground \\
\hline $\operatorname{LFI} 28 \ldots \ldots \ldots \ldots$ & Ground & Ground \\
\hline
\end{tabular}

Notes. ${ }^{(a)}$ Determined during in-flight tests; ${ }^{(b)}$ Determined during satellite-level ground tests; ${ }^{(c)}$ Determined during module-level ground tests.

compression. The ratio $\varepsilon_{\mathrm{q}} / \sigma$ can be monitored directly in-flight with the so-called "calibration channel", a telemetry mode that provides $15 \mathrm{~min}$ of uncompressed data for each diode every day (Bersanelli et al. 2010).

To limit the loss of sensitivity to less than $1 \%$, we require $\varepsilon_{\mathrm{q}} / \sigma<0.1$ in the differenced data (Maris et al. 2009). The results of the SPU calibration are summarized in Fig. 8 and Table 5. The $\left(\varepsilon_{\mathrm{q}} / \sigma\right)_{\text {dif }}<0.1$ requirement in the differenced data is satisfied by every channel.

The stability of the SPU compressor is monitored daily by an automatic procedure that checks for variations of the telemetry and quantisation error and for near-saturation conditions. $\varepsilon_{\mathrm{q}}$ has changed little during the first year of operations. Typical variations are much less than $1 \%$, with maximum variations around $1.7 \%$.

\subsection{Instrument response during cooldown}

LFI observations began during the cooldown, even before tuning. Figure 9 shows some of the first differential, uncalibrated output from LFI28M-00.

Figure 10 shows a "pseudo-map". The horizontal axis is spin axis phase angle, the vertical axis is revolution number, and the color scale gives signal amplitude. The map shows the sky, reference, and difference data for 100 telescope revolutions on 14 June 2009. These are the same data that appear in the phase binned data in Fig. 9. In the sky signal, one can see the galaxy spike, a hint of the CMB dipole, and fluctuations slower than the pointing period given by $1 / f$ noise fluctuations in the total power voltage output. In the reference load signal, one can see a significant gradient due to the rapid cooldown of the $4 \mathrm{~K}$ stage during this period, as well as the same $1 / f$ fluctuations seen in the sky signal. In the difference signal, one can see that correlated fluctuations in the sky and reference data are cancelled. The mean value has been subtracted from each revolution in the difference map.

\subsection{Operations after verification phase}

Since the beginning of nominal LFI operations, no instrument parameters have been changed, with one exception. Starting on 11 August 2009, occasional uncommanded jumps in output signal in a single channel were seen, sometimes causing saturation in the ADC and temporary loss of science data. These jumps were traced to single bit-flip changes in the DAE gain-setting circuit, presumably a result of single-event upsets (SEUs) from cosmic rays. Initially, science data were lost in saturated channels until the gain could be reset during the next downlink period. Starting in October 2009, an automatic procedure resets all gain values every $\sim 40 \mathrm{~min}$.

Figure 11 shows the cumulative number of gain change events as a function of time during the first year of operation. A total of 38 were seen through 30 September 2010, corresponding to an average rate of about one event every 11 days. Out of these 38 events, 13 saturated the ADC, leading to lost observation time from the corresponding detector, five did not saturate the ADC but the compression algorithm was not able to deal with the anomalous signal statistics, and 20 could be recovered simply by applying the correct gain after the event.

In most cases the gain increased, but occasionally it decreased. The bit flip occurs in different channels, on different bits, and the resulting value after the flip is not always the same. The most significant bit (controlling the second amplifier stage) never flips, suggesting that the component undergoing a possible single event upset (SEU) is the programmable gain amplifier in the first amplifier stage, an Analog Devices AD526SD/883B. The internal technology of the gain amplifiers in the two stages is different, therefore a different sensitivity to high energy events would not be too surprising. Nevertheless, investigation ${ }^{4}$ revealed no information on SEU effects on AD526 devices. The event rate is too low to reveal a correlation between solar activity and cosmic ray fluxes as measured by the onboard space radiation environment monitor (SREM; Planck Collaboration 2011a); however, a significant population of high energy cosmic rays, larger than expected before launch, has been observed by the HFI instrument (Planck HFI Core Team 2011a).

${ }^{4}$ Bibliographic research on Electronic Radiation Response Information Center (ERRIC), NASA GFSC Radhome; ESA radiation effect database 


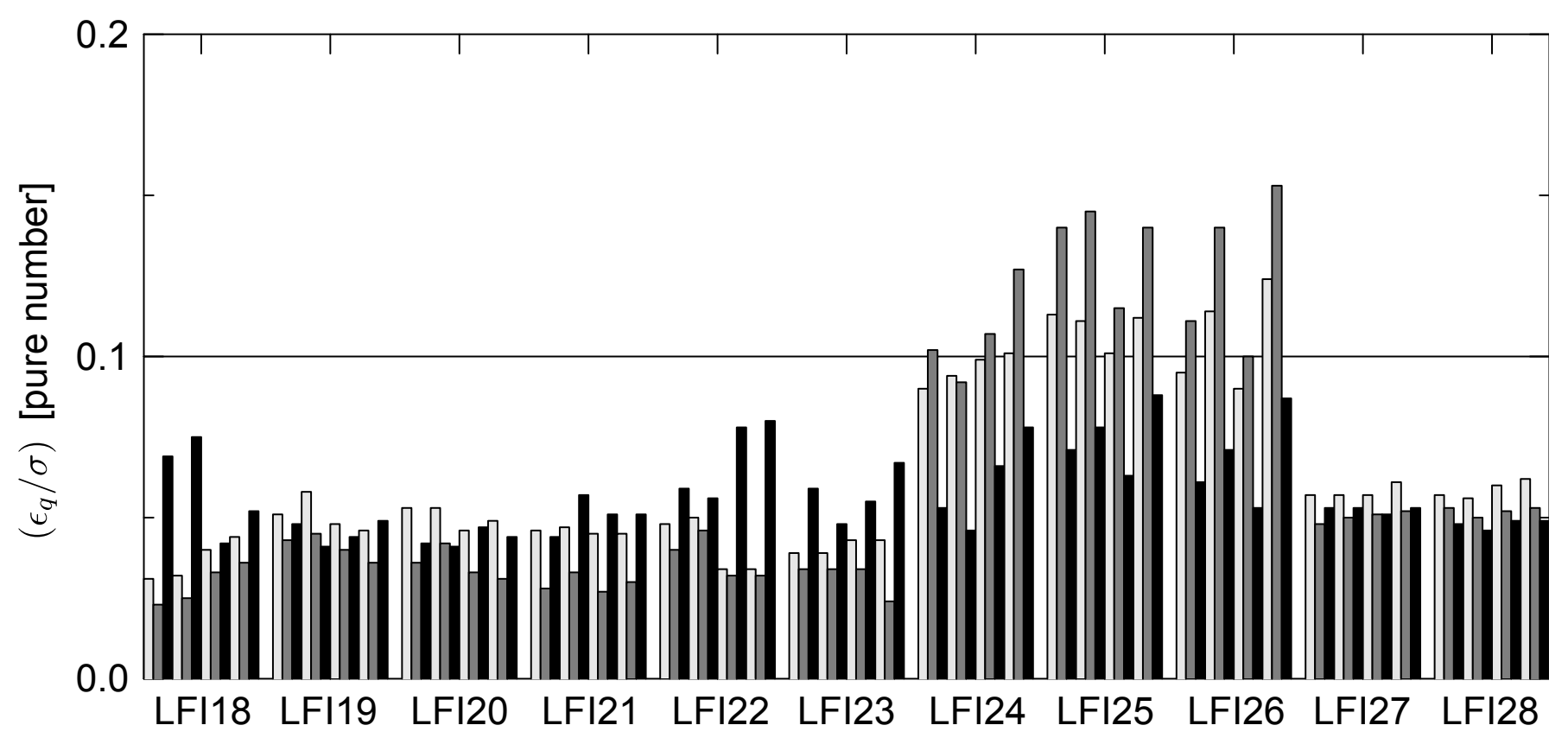

Fig. 8. Average values of $\varepsilon_{\mathrm{q}} / \sigma$ (quantisation error normalized to RMS) measured during the CPV tests for the 44 LFI detectors grouped by receiver. For each channel, three bars show $\varepsilon_{\mathrm{q}} / \sigma$ for the: (i) sky signal (white); (ii) reference load signal (gray); and (iii) differential signal (black). The $\varepsilon_{\mathrm{q}} / \sigma<0.1$ requirement in the difference data (Maris et al. 2009) is satisfied by every channel.

Table 5. Normalized quantisation error $\varepsilon_{\mathrm{q}} / \sigma$ for the $N$ channels at each frequency.

\begin{tabular}{|c|c|c|c|c|c|c|c|c|c|c|c|}
\hline & \multirow[b]{3}{*}{ FREQUENCY } & \multirow[b]{3}{*}{$N$} & \multicolumn{9}{|c|}{$\varepsilon_{\mathrm{q}} / \sigma[\%]$} \\
\hline & & & \multicolumn{3}{|c|}{ Sky } & \multicolumn{3}{|c|}{ Reference } & \multicolumn{3}{|c|}{ Difference } \\
\hline & & & Median & Min & $\operatorname{Max}$ & Median & Min & $\operatorname{Max}$ & Median & Min & $\operatorname{Max}$ \\
\hline 70 & & 24 & 4.5 & 3.1 & 5.8 & 3.3 & 2.3 & 4.6 & 5.1 & 4.1 & 8.0 \\
\hline 44 & $\cdots$ & 12 & 10.1 & 9.0 & 12.4 & 12.1 & 9.2 & 15.3 & 6.9 & 4.6 & 8.8 \\
\hline 30 & 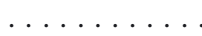 & 8 & 5.7 & 5.6 & 6.2 & 5.1 & 4.8 & 5.3 & 5.0 & 4.6 & 5.3 \\
\hline
\end{tabular}

Notes. Values are plotted in Fig. 8.

\subsection{Missing and usable data}

Since the start of the mission, only a small percentage of the LFI science data has been lost or considered unusable for science. Table 6 gives the percentage of time lost to missing data, anomalies, and maneuvers for the period from 12 August 2009 to 7 June 2010.

The main source of missing data is telemetry packets where the arithmetic compression performed by the SPU is incorrect, causing a decompression error. There were 15 such packets in all LFI channels, with negligible scientific impact. For instance, for the entire $70 \mathrm{GHz}$ frequency channel, there are just 101 lost seconds.

Anomalies include the DAE gain jumps described in Sect. 3.3, and other instabilities (see Sect. 5.1) that make the data unsuitable for science.

Spacecraft maneuvers, from routine repointings of the spin axis to stationkeeping maneuvers (see Planck Collaboration 2011a) cause by far the largest fraction of discarded data so far; however, we expect these data to be fully recovered after additional analysis of the startracker and gyroscope data. If this expectation is met, and the performance of Planck remains as it has been, well over $99 \%$ of all observing time will be usable.

\section{Beams and angular resolution}

The most accurate measurements of the LFI main beams have been made with Jupiter, the most powerful unresolved (to Planck) celestial source in the LFI frequency range. Since the LFI feed horns point to different positions on the sky, they detect the signal at different times. Table 7 gives the dates of Jupiter observations in the Fall of 2009.

The first step in extraction of the beams was to remove $1 / f$-type noise from the data using the Madam destriping mapmaking code. Planets were masked during this process. Details of the Madam destriper and of the pipeline implemented to extract beams from planet measurements are reported in Zacchei et al. (2011).

To map the beam, each sample in the selected timelines was projected in the $(u, v)$-plane perpendicular to the nominal lineof-sight (LOS) of the telescope (and at $85^{\circ}$ to the satellite spin axis). The $u$ and $v$ coordinates are defined in terms of the usual spherical coordinates $(\theta, \phi)$ :

$u=\sin \theta \cos \phi$,

$v=\sin \theta \sin \phi$.

To increase the signal-to-noise ratio, data were binned in an angular region of $2^{\prime}$ for the $70 \mathrm{GHz}$ channels and $4^{\prime}$ for the 30 and 
A. Mennella et al.: Planck early results. III.

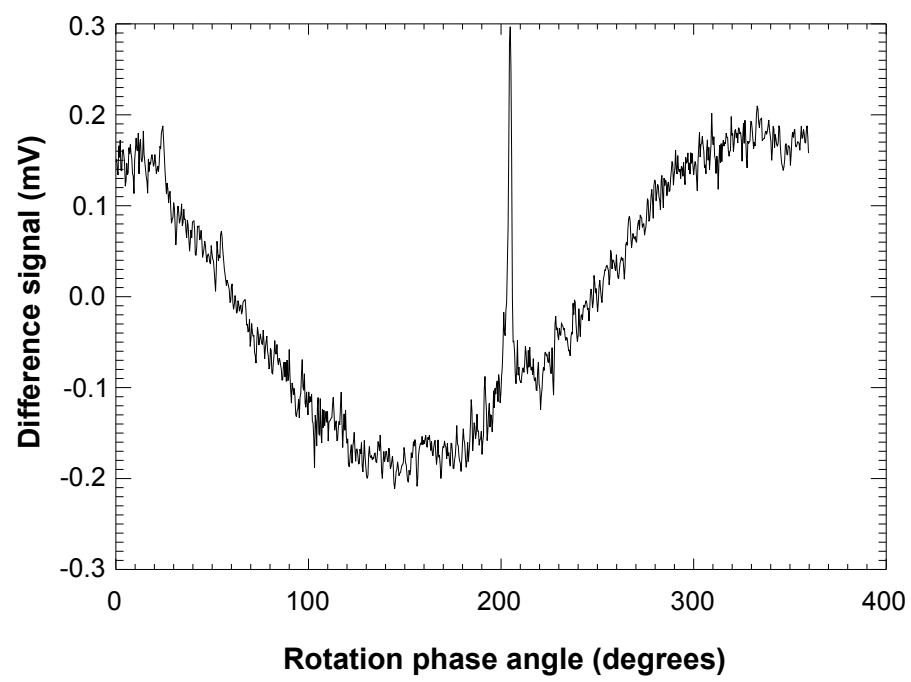

Fig. 9. Phase-binned, uncalibrated, differential data from LFI28M-00, acquired on 14 June 2009 near the beginning of the performance verification phase. Data for 100 revolutions of the telescope have been averaged over 960 "phase bins" of the rotation angle. The CMB dipole signal, which was used for preliminary photometric calibration, and a spike measured while crossing the galactic plane, are clearly visible.

Table 6. Percentages of usable and unusable data for the period 8 August 2009 to 7 June 2010.

\begin{tabular}{cccc}
\hline \hline & $30 \mathrm{GHz}$ & $44 \mathrm{GHz}$ & $70 \mathrm{GHz}$ \\
Category & {$[\%]$} & {$[\%]$} & {$[\%]$} \\
\hline Missing . . . . . . . . & $1.6 \times 10^{-4}$ & $2.7 \times 10^{-4}$ & $3.9 \times 10^{-4}$ \\
Anomalies . . . . . . . . . . . . . & 0.4 & 0.7 & 0.4 \\
Maneuvers . . & 91.3 & 8.3 & 8.3 \\
Used . . . $\ldots 1.0$ & 91.3 \\
\hline
\end{tabular}

Notes. See text for explanation of the categories.

$44 \mathrm{GHz}$ channels. We recovered all beams down to $-20 \mathrm{~dB}$ from the peak. An elliptical Gaussian was fit to each beam for both $\mathrm{M}$ and $\mathrm{S}$ radiometers. The FWHM given in Table 8 is the square root of the product of the major axis and minor axis FWHMs of the individual beams, averaged between $\mathrm{M}$ and $\mathrm{S}$ radiometers. The uncertainties in Table 8 are the standard deviation of the mean of the $1 \sigma$ statistical uncertainties of the fit. Differences between the $\mathrm{M}$ and $\mathrm{S}$ beams caused by optics and receiver nonidealities are inevitable at some level, but they appear to be well within the statistical uncertainties, and for the purposes of pointsource extraction relevant for this paper, the beams may be considered identical. Table 8 gives the typical FWHM and ellipticity averaged over each frequency channel. Exhaustive details on all LFI beam parameters will be presented in the future.

Figure 12 shows three examples of measured beams compared with calculations performed with the GRASP9 $9^{5}$ software. The calculated beams have been smeared appropriately to take into account satellite rotation and sampling. The effect of sampling is evident already at $-3 \mathrm{~dB}$ and cannot be neglected (the typical effect on FWHM is $2 \%$ at $70 \mathrm{GHz}$ ). In the comparison of measured and calculated beams, the peak of the simulated

\footnotetext{
5 http://www.ticra.com
}

Table 7. Dates of Jupiter observations in 2009.

\begin{tabular}{|c|c|c|}
\hline RCA & Operational day & Date \\
\hline \multicolumn{3}{|l|}{$70 \mathrm{GHz}$} \\
\hline 18,23 & $168-169$ & 28 October-29 October \\
\hline 19,22 & $169-170$ & 29 October-30 October \\
\hline 18,23 & $169-171$ & 29 October-31 October \\
\hline \multicolumn{3}{|l|}{$44 \mathrm{GHz}$} \\
\hline 24 & $170-171$ & 30 October-31 October \\
\hline 25,26 & $163-165$ & 23 October-25 October \\
\hline \multicolumn{3}{|l|}{$30 \mathrm{GHz}$} \\
\hline 27,28 & $170-172$ & 30 October-1 November \\
\hline
\end{tabular}

beam was aligned with the peak of the measured beam (calculated from a Gaussian elliptical fit). The electromagnetic model of the design telescope (Sandri et al. 2010) was used as a reference in the comparison. Ideal parameters have been assumed for the shape of the mirrors, the alignment of the telescope and focal plane unit, as well as for the pattern of the feed horns. The good agreement down to $-20 \mathrm{~dB}$ demonstrates that, to first order, the overall LFI optical system is performing as expected. Comparison with a more realistic telescope model, including the actual alignment, measured mirror shapes, and measured feedhorn patterns, will be considered in future work.

\section{Stability and calibration}

\subsection{Stability}

Thanks to its differential scheme, the LFI is insensitive to many effects caused by $1 / f$ noise, thermal fluctuations, or electrical instabilities.

One effect detected during the first survey was the daily temperature fluctuation in the back-end unit induced by the downlink transponder, which was powered on each day for downlinks during the first 258 days of the mission (Planck Collaboration 2011b, Sects. 2.4.1 and 5.1). Figure 13 shows the effect of these fluctuations on the radiometeric output of LFI27M. As expected, the effect is highly correlated between the sky and reference load signals. In the difference, the variation is reduced by a factor $\sim(1-r)$, where $r$ is the gain modulation factor defined in Eq. (3).

A particular class of signal fluctuations occasionally observed during operations is due to electrical instabilities that appear as abrupt increases in the measured drain current of the front-end amplifiers, with a relaxation time variable from few seconds to some hundreds of seconds. Typically, these events cause a simultaneous change in the sky and reference load signals. Because they are essentially common-mode, their residual on the differenced data is negligible (Fig. 14), and the data are suitable for science production. In a few cases the residual fluctuation in the differential output was large enough (a few millikelvin in calibrated antenna temperature units) to be flagged, and the data were not used. The total amount of discarded data for all LFI channels until Operational Day 389 was about 2000 s per detector, or $0.008 \%$.

A further peculiar effect appeared in the $44 \mathrm{GHz}$ detectors, where single isolated samples, either on the sky or the reference voltage output, were far from the rest. Over a reference period of four months, 15 occurrences of single-sample spikes (out of 


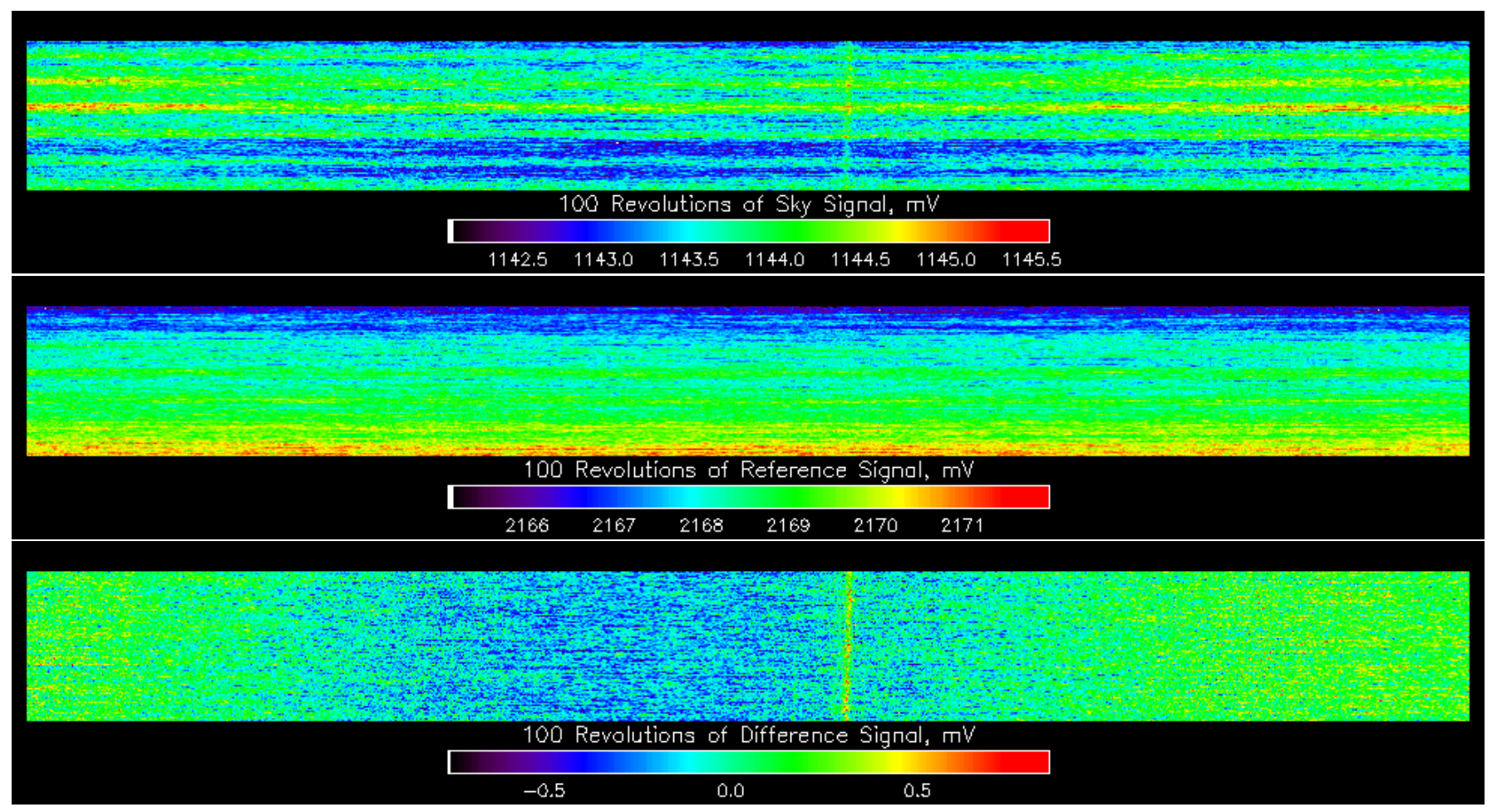

Fig. 10. Phase binned uncalibrated pseudo-maps for LFI28M-00. Top: sky signal. Middle: reference load signal. Bottom: difference signal. The horizontal axis is spin axis phase angle, the vertical axis is revolution number, and the color scale gives signal amplitude. The mean value has been subtracted from each revolution in the difference map.

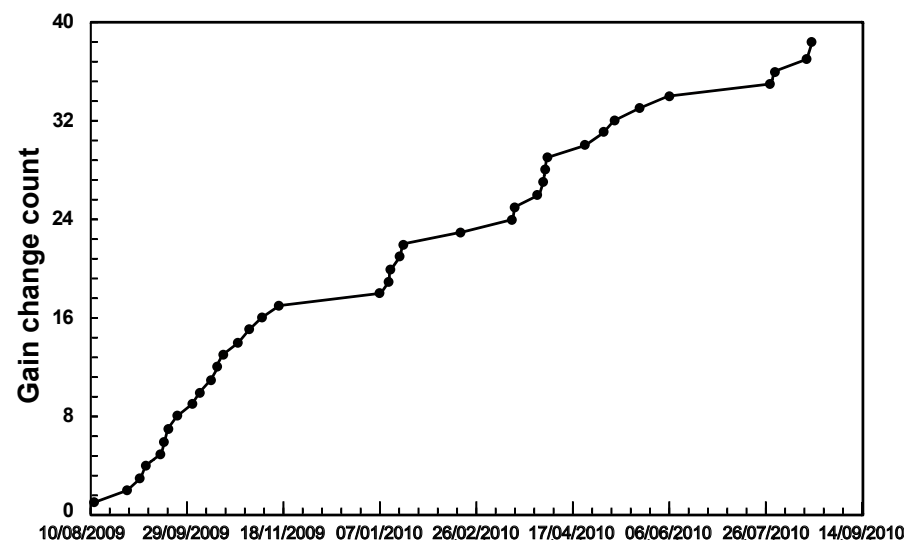

Fig. 11. Cumulative number of gain change events during the first year of operations.

24 total anomaly events) were discarded, an insignificant loss of data.

\subsection{Calibration}

Photometric calibration, i.e., conversion from voltage to antenna temperature, is performed for each radiometer after total power data have been cleaned of $1 \mathrm{~Hz}$ frequency spikes (see Sect. 7 and Zacchei et al. 2011), and differenced.

Our calibrator is the well-known dipole signal induced by Earth and spacecraft motions with respect to the CMB rest frame. The largest calibration uncertainty comes from the presence of the Galaxy and of the CMB anisotropies in the
Table 8. LFI beam FWHM and mean ellipticity measured in flight from the first Jupiter pass.

\begin{tabular}{rccc}
\hline \hline RCA & $\begin{array}{c}\text { FWHM }^{a} \\
{\left[^{\prime}\right]}\end{array}$ & $\begin{array}{c}\text { Uncertainty }^{b} \\
{\left[^{\prime}\right]}\end{array}$ & Ellipticity $^{c}$ \\
\hline $\mathbf{7 0 ~ G H z ~ a v e r a g e ~} \ldots \ldots$ & $\mathbf{1 3 . 0 1}$ & & $\mathbf{1 . 2 7}$ \\
LFI18 $\ldots \ldots \ldots$ & 13.39 & 0.170 & \\
LFI19 $\ldots \ldots \ldots \ldots$ & 13.01 & 0.174 & \\
LFI20 $\ldots \ldots \ldots$ & 12.75 & 0.170 & \\
LFI21 $\ldots \ldots \ldots$ & 12.74 & 0.156 & \\
LFI22 $\ldots \ldots \ldots$ & 12.87 & 0.164 & \\
LFI23 $\ldots \ldots \ldots$ & 13.27 & 0.171 & \\
$\mathbf{4 4 ~ G H z ~ a v e r a g e ~} \ldots \ldots$ & $\mathbf{2 7 . 9 2}$ & & $\mathbf{1 . 2 6}$ \\
LFI24 $\ldots \ldots \ldots$ & 22.98 & 0.652 & \\
LFI25 $\ldots \ldots \ldots$ & 30.46 & 1.075 & \\
LFI26 $\ldots \ldots \ldots$ & 30.31 & 1.131 & \\
$\mathbf{3 0 ~ G H z ~ a v e r a g e ~} \ldots \ldots$ & $\mathbf{3 2 . 6 5}$ & & $\mathbf{1 . 3 8}$ \\
LFI27 $\ldots \ldots \ldots$ & 32.65 & 1.266 & \\
LFI28 $\ldots \ldots \ldots$ & 32.66 & 1.287 & \\
\hline
\end{tabular}

Notes. ${ }^{(a)}$ The square root of the product of the major axis and minor axis FWHMs of the individual RCA beams, averaged between $\mathrm{M}$ and $\mathrm{S}$ radiometers; ${ }^{(b)}$ the standard deviation of the mean of the $1 \sigma$ statistical uncertainties of the fit. Although a small difference between the $M$ and $\mathrm{S}$ beams caused by optics and receiver non-idealities can be expected, for the purpose of point-source extraction relevant for this paper the beams are considered identical, as this effect is well within the statistical uncertainty; ${ }^{(c)}$ ratio of the major and minor axes of the fitted elliptical Gaussian.

measured signal. We therefore use an iterative calibration procedure in which the dipole is fitted and subtracted, producing a 

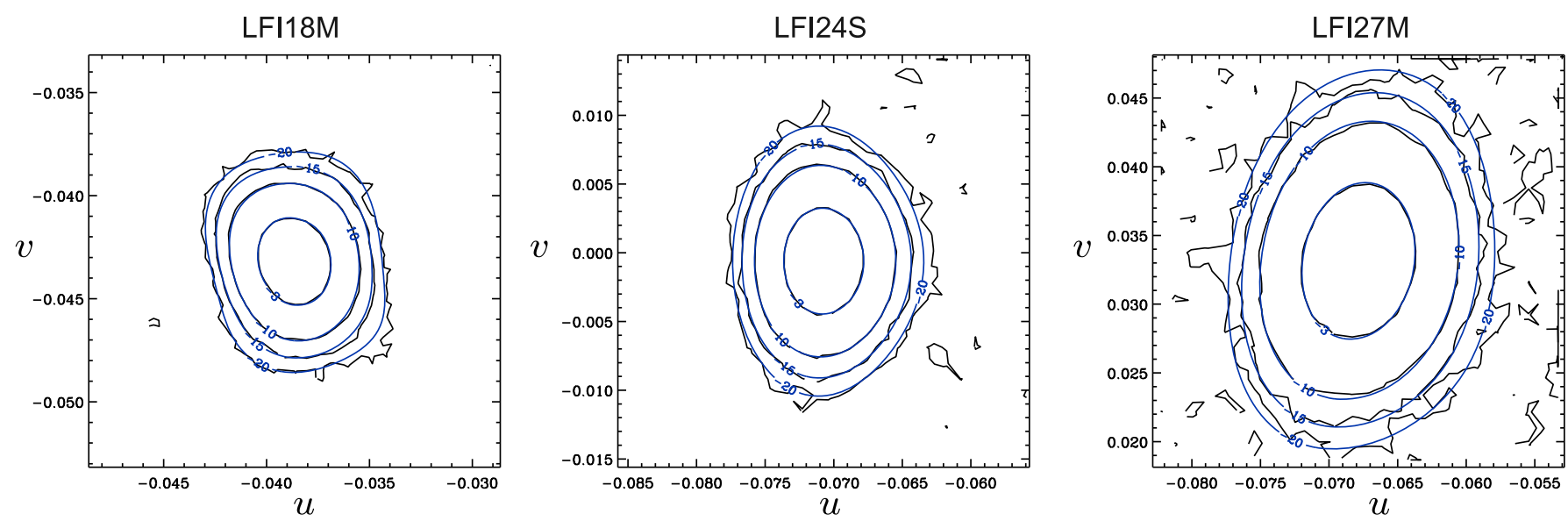

Fig. 12. Examples (one per frequency channel) of the LFI measured beams compared with simulations. The simulated main beams have been computed in the co- and cross-polar basis according to Ludwig's third definition (Ludwig 1973), in spherical grids with $301 \times 301$ points defined with respect to the LOS frame. They are referred to the design telescope configuration. In each plot the contours are the levels at $-3,-10,-15$, and $-20 \mathrm{~dB}$ from the corresponding power peak. The simulations have been carried out in the transmitting mode using GRASP9 software. Physical optics and physical theory of diffraction have been used on both reflectors.
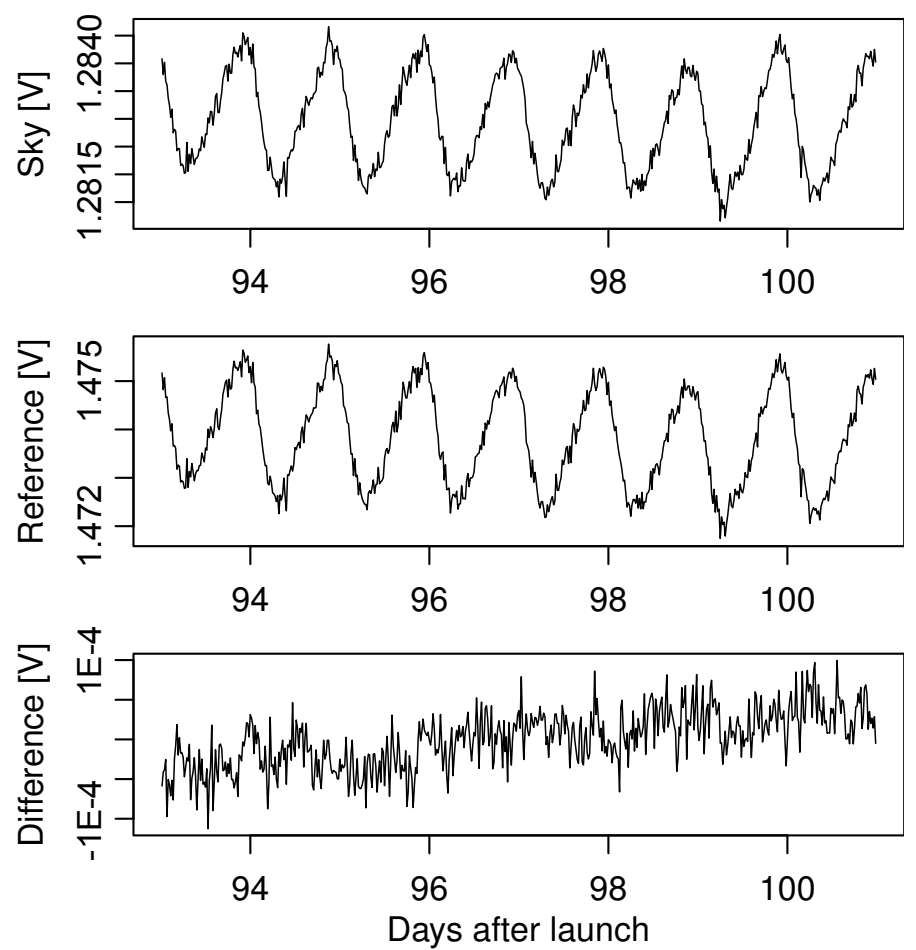

Fig. 13. Sky (top), reference (middle) and difference (bottom) signals from radiometer LFI27M. The modulation is due to the thermal effect induced by the satellite transponder being turned on and off for the daily downlink early in the mission. The quasi-sinusoidal $\sim 0.1 \%$ fluctuation in the sky and reference signals is almost completely removed in the differenced data.

sky map that is then removed from the original data to enhance the dipole signal for the next iteration. Typically, convergence is obtained after a few tens of iterations.

The thin gray line in Fig. 15 shows the result of this iterative process for the radiometer LFI21M, pointing period by pointing period. In the most stable regions the gain values display relative variations of $\sim 0.8 \% \mathrm{rms}$ and $\sim 7 \%$ peak-to-peak. In the most unstable region, where the spacecraft spin was nearly aligned with the dipole and the dipole signal was weak, the relative variations are $\sim 4 \% \mathrm{rms}$ and $\sim 67 \%$ peak-to-peak. These variations reflect statistical uncertainties in the determination of the gain over a single pointing period, rather than actual changes in gain.

We can put a limit on the true intrinsic gain variations by looking at the variation of the total power voltage output (cf. Sect. 5.1). The small variations in total power constrain intrinsic gain variations to be less than $1 \%$.

The gain solution based on individual pointing periods was therefore processed to improve its stability by implementing two running averages that have been further smoothed with wavelets, with a 5-day window in strong dipole regions and a 30-day window in weak dipole regions. In particular cases, where a known instrument gain change could be identified $^{6}$, we used a 5 -day un-smoothed window. In Fig. 15 the thick black line gives the smoothed gain curve; variations are now $\sim 0.5 \% \mathrm{rms}$ and $\sim 1.6 \%$ peak-to-peak. The inset compares the smoothed gain and the relative gain variation obtained from Eq. (16), which represent true gain fluctuations in the instrument at the level of $\pm 0.2 \%$. In the current gain model implementation these changes are neglected, but they will be incuded in future versions of our analysis.

\subsection{Calibration accuracy}

Following COBE and WMAP (Kogut et al. 1996; Hinshaw et al. 2009; Jarosik et al. 2011), our main calibrator is the dipole modulation in the CMB. In our current calibration model we use as calibration signal the sum of the solar dipole $\Delta T_{\text {Sun }}$ and the orbital dipole $\Delta T_{\text {orb }}$, which is the contribution from Planck's orbital velocity around the Sun,

$\Delta T=\left(\Delta T_{\mathrm{Sun}}+\Delta T_{\mathrm{orb}}\right) \sin \vartheta_{\mathrm{axis}}$,

where $\vartheta_{\text {axis }}$ is the angle between the spacecraft axis and the overall dipole axis (solar + orbital).

In Eq. (11), the absolute calibration uncertainty is dominated by the uncertainty in $\Delta T_{\text {Sun }}=3.355 \mathrm{mK} \pm 0.008 \mathrm{mK}$ (Hinshaw et al. 2009), which is known to about $0.2 \%$. The modulation of the orbital dipole by the Earth motion around the Sun is known

\footnotetext{
6 E.g., when the transponder was powered on continuously, which caused a change in the instrument warm unit temperature.
} 

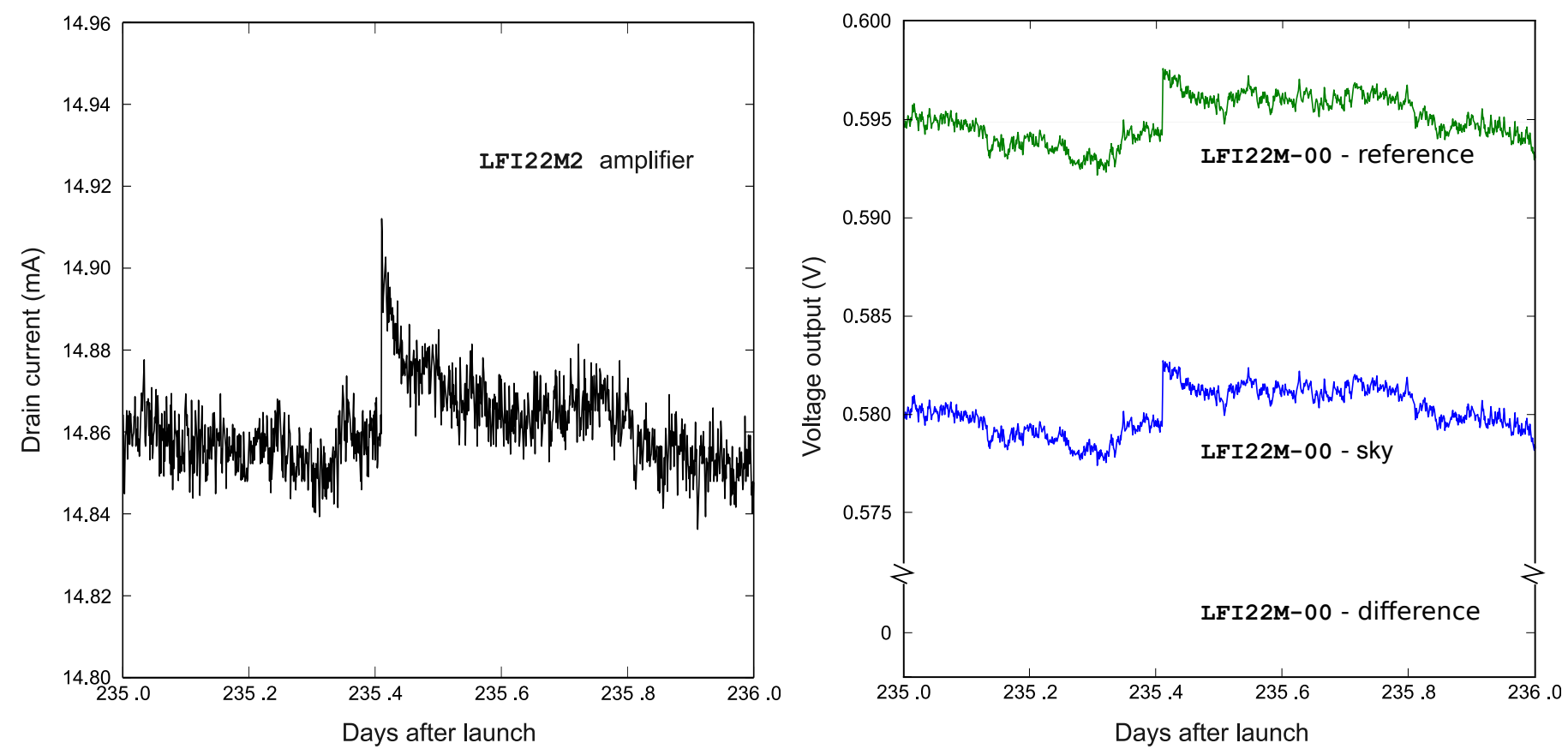

Fig. 14. Short spikes in the drain current (left) affect total power signals (right). The jumps are strongly correlated in sky and reference signals, so that in the difference data the effect essentially disappears.

with an uncertainty almost three orders of magnitude smaller; however, at least one complete Planck orbit is needed for its measurement. In the future, calibration based on the Earth orbital modulation will be significantly more accurate.

The accuracy of our current calibration can be estimated by taking into account two components: 1) the statistical uncertainty in the regions of weak dipole; and 2) the systematic uncertainty caused by neglecting gain fluctuations that occur on periods shorter than the smoothing window. In our calibration procedure the gain is estimated for every pointing period: if we call $G_{i}$ the gain estimate from the $i$ th pointing period we have that the associated uncertainty is

$\delta G=\sqrt{\frac{\sum_{i=1}^{N}\left(G_{i}-\langle G\rangle\right)^{2}}{N-1}}$,

where $N$ is the overall number of pointings and $\langle G\rangle$ is the average of the $N$ gains. We then approximate the effect of the smoothing filter as an average over $M$ consecutive pointings, so that the overall uncertainty can be estimated as

$\left.\delta G\right|_{\text {stat }} \simeq \frac{\delta G}{\sqrt{M}}=\frac{1}{\sqrt{M}} \sqrt{\frac{\sum_{i=1}^{N}\left(G_{i}-\langle G\rangle\right)^{2}}{N-1}}$.

Table 9 lists the largest statistical uncertainties in four time windows (days 100-140, 280-320, 205-245, 349-389), the first two corresponding to minimum and the second two to maximum dipole response. In order to provide conservative estimates we have always chosen a value for $M$ corresponding to the number of pointings in 5 days also in the cases where a 30-day smoothing window was used.

Our current calibration scheme neglects systematic gain variations caused by thermal fluctuations, which introduce spurious signal fluctuations on timescales ranging from 1 to $24 \mathrm{~h}$. Being much slower than the satellite spin period, these fluctuations are well-removed during map-making by the destriping algorithm
Table 9. Worst-case relative calibration uncertainties.

\begin{tabular}{|c|c|c|}
\hline \multirow[b]{2}{*}{ RCA } & \multicolumn{2}{|c|}{$\delta G /\left.G\right|_{\text {stat }}[\%]$} \\
\hline & Rad. M & Rad. S \\
\hline $70 \mathrm{GHz}$ average $\ldots \ldots \ldots$ & \multicolumn{2}{|c|}{0.12} \\
\hline $\begin{array}{l}\text { LFI18 } \ldots \ldots \ldots \\
\text { LFI19 } \ldots \ldots \ldots \\
\text { LFI20 } \ldots \ldots \ldots \ldots \\
\text { LFI21 } \ldots \ldots \ldots \\
\text { LFI22 } \ldots \ldots \ldots \\
\text { LFI23 } \ldots \ldots \ldots \ldots\end{array}$ & $\begin{array}{l}0.28 \\
0.14 \\
0.25 \\
0.37 \\
0.37 \\
0.29\end{array}$ & $\begin{array}{l}0.24 \\
0.23 \\
0.26 \\
1.04 \\
0.18 \\
0.38\end{array}$ \\
\hline $44 \mathrm{GHz}$ average $\ldots \ldots \ldots$ & \multicolumn{2}{|c|}{0.07} \\
\hline $\begin{array}{l}\operatorname{LFI} 24 \ldots \ldots \ldots \ldots \\
\text { LFI25 } \ldots \ldots \ldots \ldots \\
\text { LFI26 } \ldots \ldots \ldots \ldots\end{array}$ & $\begin{array}{l}0.35 \\
0.36 \\
0.40\end{array}$ & $\begin{array}{l}0.31 \\
0.30 \\
0.41\end{array}$ \\
\hline $\begin{array}{r}30 \text { GHz average } \ldots \ldots \ldots \\
\text { LFI } 27 \ldots \ldots \ldots \ldots \\
\text { LFI } 28 \ldots \ldots \ldots \ldots\end{array}$ & $\begin{array}{l}0.31 \\
0.23\end{array}$ & $\begin{array}{l}0.35 \\
0.38\end{array}$ \\
\hline
\end{tabular}

(Keihänen et al. 2005, 2010; Zacchei et al. 2011), so that the rms systematic uncertainty per pixel in the final maps due to imperfect calibration is at sub-microkelvin levels (see Sect. 7.2). However, we expect that a more accurate description of the gain variations over short timescales will improve our calibration accuracy even at the level of time ordered data. If we use the dipole signature to estimate an average value $G_{0}$ for the gain over long time periods (e.g., several months), we can write the gain versus time as

$G(t)=G_{0} \times(1+\xi(t))$,

where $\xi(t) \equiv \delta G /\langle G\rangle$ is the relative gain variation.

The simplest and most direct measurement of gain changes is through the monitoring of the stability of the reference load 


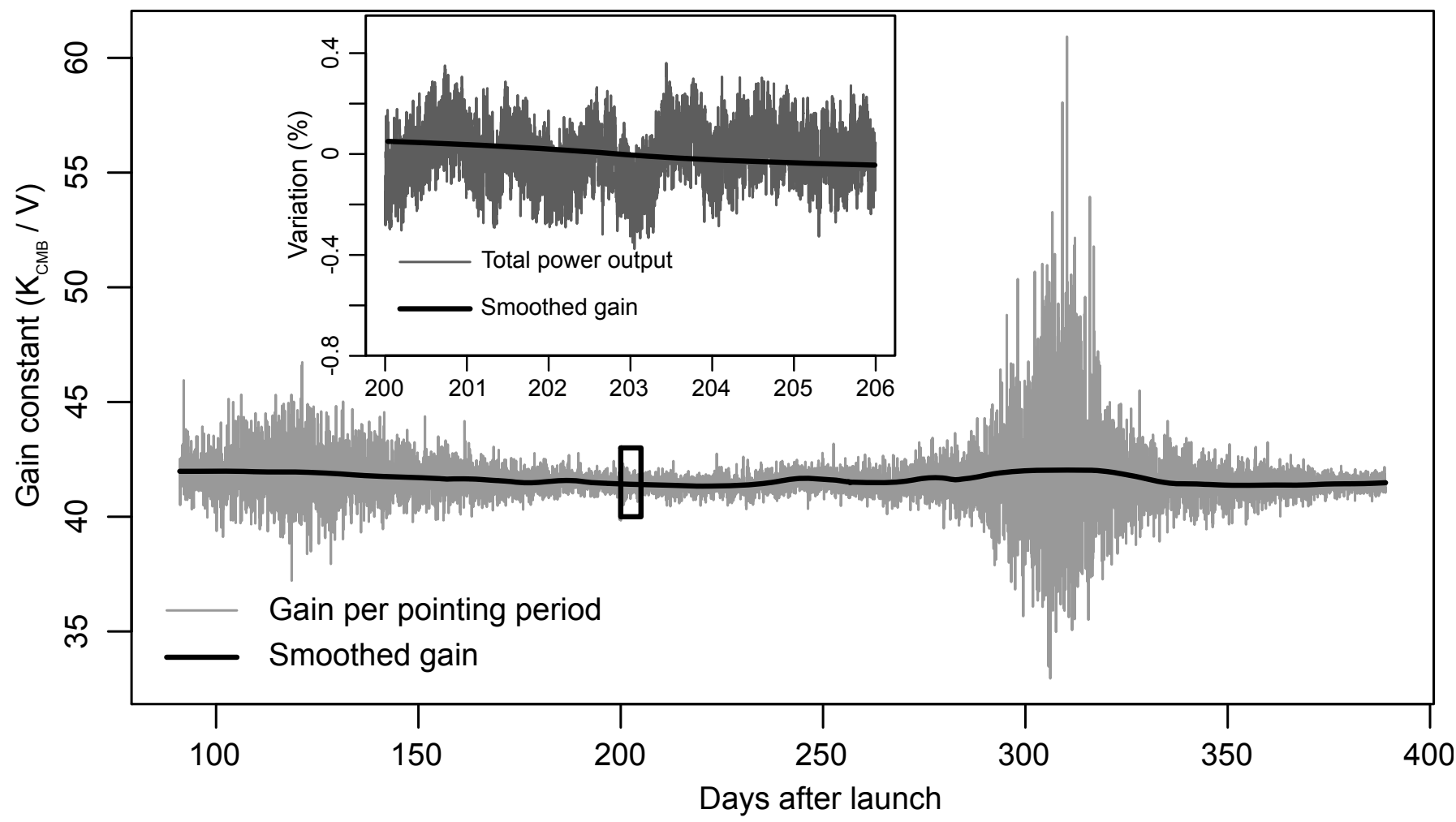

Fig. 15. Reconstructed gain for radiometer LFI21M. Thin gray line: gain constants obtained for each pointing period by the iterative calibration procedure. The two regions showing large scatter in the reconstructed gain correspond to periods with minimum amplitude in the CMB dipole. Thick black line: gain constants obtained after smoothing - this is the actual curve used in the pipeline. The inset shows a closer look at the region between Operational Days 200 and 205. Here the relative variation for the smoothed gain model is compared with the relative gain variation calculated from Eq. (16).

total power voltage output, $V_{\text {ref }}=K\left(T_{\text {ref }}+\epsilon_{\text {iso }} T_{\text {sky }}+T_{\text {noise }}\right)$, where $K=1 / G$ and $\epsilon_{\text {iso }}$ is the "isolation", i.e., the percentage of sky signal leaking into the reference load signal because of non-idealities in the front-end modules, such as amplitude and phase imbalances in the LNAs and phase switches. The relative output voltage variation is:

$\frac{\delta V_{\text {ref }}}{\left\langle V_{\text {ref }}\right\rangle}=\left[\left(\frac{\delta K}{\langle K\rangle}\right)^{2}+\left(\frac{\delta T_{\text {ref }}}{\left\langle T_{\text {sys }}\right\rangle}\right)^{2}+\left(\frac{\epsilon_{\text {iso }} \delta T_{\text {sky }}}{\left\langle T_{\text {sys }}\right\rangle}\right)^{2}+\left(\frac{\delta T_{\text {noise }}}{\left\langle T_{\text {sys }}\right\rangle}\right)^{2}\right]^{1 / 2}$,

where $T_{\text {sys }}=T_{\text {ref }}+\epsilon_{\text {iso }} T_{\text {sky }}+T_{\text {noise }}$.

Consider the four terms on the right-hand side of Eq. (15). The first, $(\delta K /\langle K\rangle)^{2}$, gives the effect of gain variations. To estimate the second, $\left(\delta T_{\text {ref }} /\left\langle T_{\text {sys }}\right\rangle\right)^{2}$, we know that $T_{\text {noise }}$ is in the range $10-30 \mathrm{~K}$ and the reference load temperature is about $4.5 \mathrm{~K}$ with fluctuations of $\lesssim 2 \mathrm{mK}$. So $\delta T_{\text {ref }} /\left\langle T_{\text {sys }}\right\rangle<2 \times 10^{-4}$. For the third, with upper limits in the isolation and sky signal variations given by $\epsilon_{\text {iso }} \lesssim 0.1$ (Mennella et al. 2010) and $\delta T_{\text {sky }} \lesssim 3.5 \mathrm{mK}$ (CMB dipole), the contribution from the sky is bounded by $\epsilon_{\text {iso }} \delta T_{\text {sky }} /\left\langle T_{\text {sys }}\right\rangle<4 \times 10^{-5}$. Finally, fluctuations in the noise temperature due to thermal changes can be estimated by assuming a coupling of $0.5 \mathrm{~K} \mathrm{~K}^{-1}$ and taking a typical temperature fluctuation of $1 \mathrm{mK}$ in the front-end unit, giving $\delta T_{\text {noise }} /\left\langle T_{\text {sys }}\right\rangle<5 \times 10^{-4}$.

Thus any variation in the total power signal larger than $\sim 6 \times 10^{-4}$ must be due to actual changes in the gain. In practice, the last three terms on the right-hand side of Eq. (15) can be neglected compared to $\delta K /\langle K\rangle$, and we have

$\frac{\delta V_{\text {ref }}}{\left\langle V_{\text {ref }}\right\rangle} \approx \frac{\delta K}{\langle K\rangle}=-\xi(t)$.

Figure 16 shows two estimates for $\xi(t)$ based on the temperature sensors in the radiometer back-end and on the relative variation of the reference load voltage output (black line).

In future versions of the LFI gain model, we plan to use the iterative solution as a starting point and then trace gain changes down to short time scales both by using housekeeping information and by monitoring the relative variation given by Eq. (16).

\section{Noise properties}

The noise characteristics of the LFI datastreams are closely reproduced by a simple (white $+1 / f$ ) noise model,

$P(f)=\sigma^{2}\left[1+\left(\frac{f}{f_{\text {knee }}}\right)^{\alpha}\right]$,

where $P(f)$ is the power spectrum and $\alpha \approx-1$.

In this model, noise properties are characterised by three parameters, the white noise limit $\sigma$, the knee frequency ${ }^{7} f_{\text {knee }}$, and the exponent of the $1 / f$ component $\alpha$, also referred to as slope. In this section we show how the LFI noise has been characterised in flight, and we give noise performace estimates based on one year of operations.

7 The frequency at which the $1 / f$ and white noise contribute equally in power. 


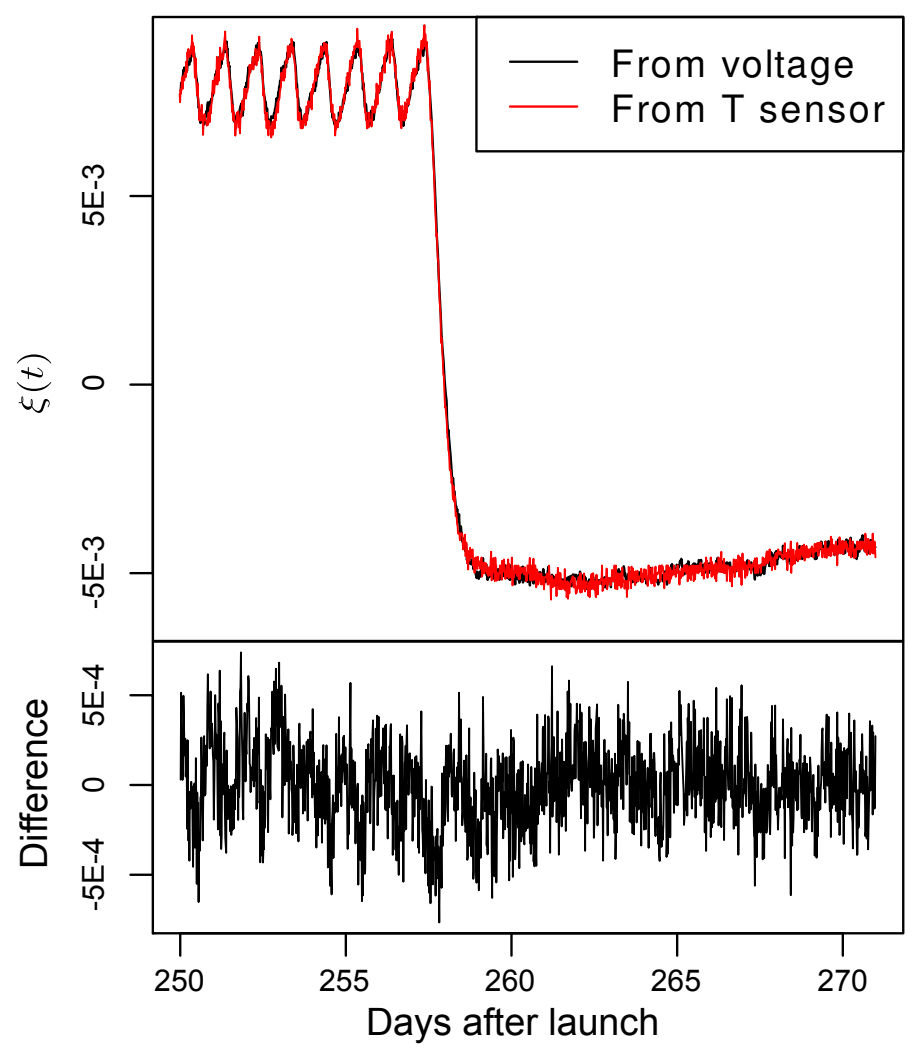

Fig. 16. Comparison between two gain models for radiometer LFI26S. Each model uses the average gain calculated using the dipole, but modulates it over time using either Eq. (16) (black line) or one of the temperature sensors in the LFI back-end (red line). The bottom plot shows the difference between the two gain models.

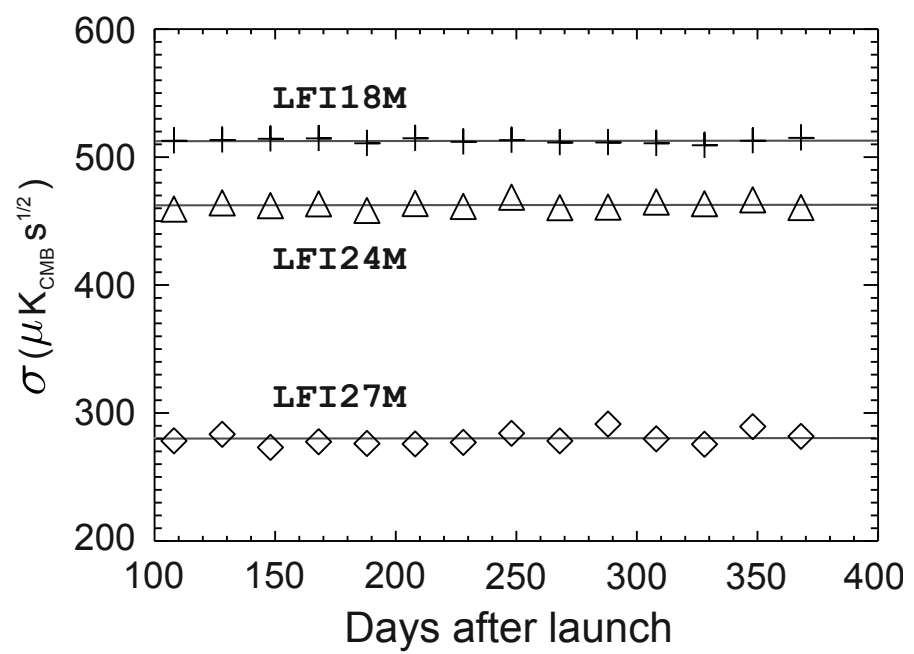

Fig. 17. White noise level versus time for three radiometers: LFI18M, LFI24M, and LFI27M.

\subsection{Method}

Noise properties have been calculated following two different and complementary approaches: 1) fitting Eq. (17) to timeordered data for each radiometer; and 2) building normalised noise maps by differencing data from the first half of the pointing period with data from the second half of the pointing period to remove the sky signal ("jackknife" data sets).
Table 10. White noise sensitivities for the LFI radiometers.

\begin{tabular}{|c|c|c|c|}
\hline \multirow{2}{*}{\multicolumn{2}{|c|}{ RCA }} & \multicolumn{2}{|c|}{ White NoISE SENSITIVITY } \\
\hline & & $\begin{array}{c}\text { Radiometer M } \\
{\left[\mu \mathrm{K}_{\mathrm{CMB}} \mathrm{s}^{1 / 2}\right]}\end{array}$ & $\begin{array}{l}\text { Radiometer S } \\
{\left[\mu \mathrm{K}_{\mathrm{CMB}} \mathrm{s}^{1 / 2}\right]}\end{array}$ \\
\hline \multicolumn{4}{|l|}{$70 \mathrm{GHz}$} \\
\hline $\begin{array}{l}\text { LFI18 } \\
\text { LFI19 } \\
\text { LFI20 } \\
\text { LFI21 } \\
\text { LFI22 } \\
\text { LFI23 }\end{array}$ & 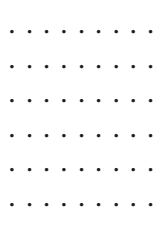 & $\begin{array}{l}512.0 \pm 1.8 \\
581.4 \pm 1.6 \\
590.8 \pm 1.7 \\
455.2 \pm 2.8 \\
492.0 \pm 2.3 \\
507.7 \pm 1.7\end{array}$ & $\begin{array}{l}465.7 \pm 1.7 \\
555.6 \pm 2.4 \\
623.2 \pm 1.5 \\
564.1 \pm 6.2 \\
534.4 \pm 2.8 \\
542.4 \pm 2.6\end{array}$ \\
\hline \multicolumn{4}{|l|}{$44 \mathrm{GHz}$} \\
\hline $\begin{array}{l}\text { LFI24 } \\
\text { LFI25 } \\
\text { LFI26 }\end{array}$ & $\begin{array}{l}\ldots \ldots \\
\cdots \ldots \ldots \\
\cdots \ldots \ldots\end{array}$ & $\begin{array}{l}462.2 \pm 1.9 \\
413.6 \pm 3.6 \\
478.6 \pm 3.1\end{array}$ & $\begin{array}{l}399.2 \pm 1.3 \\
392.6 \pm 1.6 \\
418.6 \pm 4.2\end{array}$ \\
\hline \multicolumn{4}{|l|}{$30 \mathrm{GHz}$} \\
\hline $\begin{array}{l}\text { LFI27 } \\
\text { LFI28 }\end{array}$ & $\ldots \ldots \ldots$ & $\begin{array}{l}277.7 \pm 2.1 \\
312.3 \pm 1.7\end{array}$ & $\begin{array}{l}302.9 \pm 1.6 \\
285.3 \pm 1.4\end{array}$ \\
\hline
\end{tabular}

\subsubsection{Noise estimation in the frequency domain}

Estimation of noise power spectra from time ordered data is part of the iterative approach in the ROMA map-making suite (Prunet et al. 2001; Natoli et al. 2001; de Gasperis et al. 2005). We produced joint estimates for both signal (i.e., maps) and noise, and then fitted the resulting noise power spectra to Eq. (17) to estimate the three parameters $\sigma, f_{\text {knee}}$, and $\alpha$. Pipeline details are given in Zacchei et al. (2011).

To verify the instrument stability, we produced noise estimates from five-day data chunks separated by 20 days each. Figure 17 gives examples for three radiometers. No significant systematic variations were observed in any of the three parameters, so that final values and uncertainties could be obtained by taking the average and the standard deviation of the five-day values.

Results for $\sigma, f_{\text {knee }}$, and $\alpha$ are given in Tables 10 and 11 . Typical uncertainties are $\sim 0.5 \%$ for the white noise, between 5 and $10 \%$ for the slope, and between 10 and $20 \%$ for the knee frequency. Comparing the noise power spectra for all 22 LFI radiometers with their best-fit model, we found good agreement as shown in Fig. 18. In the figure, the horizontal red lines represent the white noise level (lower line) and the level of equal contribution from white and $1 / f$ noise. The frequency corresponding to the intercept of the upper red line with the power spectrum is the knee frequecy $f_{\text {knee }}$.

\subsubsection{Jackknife noise maps}

During each pointing period Planck repeatedly scans essentially the same circle in the sky. This provides a powerful method to remove the sky signal and produce maps containing only the instrumental noise (both uncorrelated and correlated on timescales shorter than about $20 \mathrm{~min}$ ). The details of this method are given in Zacchei et al. (2011). These jackknife noise maps can be normalised to the white noise estimate at each pixel obtained from the white noise covariance matrix, so that a perfectly white noise map would be Gaussian and isotropic with unit variance. Figure 19 shows an example of such a normalised noise map for the $30 \mathrm{GHz}$ channel. The map is structureless, as expected, apart 
Table 11. Knee frequency and slope for the LFI radiometers.

\begin{tabular}{|c|c|c|c|c|}
\hline \multirow[b]{2}{*}{ RCA } & \multicolumn{2}{|c|}{ KNEE FREQUENCY } & \multicolumn{2}{|c|}{ SLOPE } \\
\hline & $\begin{array}{c}\text { Radiometer M } \\
{[\mathrm{mHz}]}\end{array}$ & $\begin{array}{c}\text { Radiometer S } \\
{[\mathrm{mHz}]}\end{array}$ & Radiometer M & Radiometer S \\
\hline \multicolumn{5}{|l|}{$70 \mathrm{GHz}$} \\
\hline $\begin{array}{l}\text { LFI18 } \\
\text { LFI19 } \\
\text { LFI20 } \\
\text { LFI21 } \\
\text { LFI22 } \\
\text { LFI23 }\end{array}$ & $\begin{array}{l}16.3 \pm 3.9 \\
15.1 \pm 2.2 \\
18.7 \pm 5.3 \\
37.2 \pm 6.1 \\
12.7 \pm 19.7 \\
34.6 \pm 4.8\end{array}$ & $\begin{array}{r}17.7 \pm 2.5 \\
22.0 \pm 5.0 \\
8.7 \pm 1.6 \\
25.9 \pm 7.9 \\
15.8 \pm 6.2 \\
129.8 \pm 6.9\end{array}$ & $\begin{array}{l}-1.04 \pm 0.08 \\
-1.09 \pm 0.05 \\
-0.69 \pm 0.07 \\
-1.56 \pm 0.06 \\
-1.01 \pm 0.15 \\
-0.96 \pm 0.04\end{array}$ & $\begin{array}{l}-1.15 \pm 0.07 \\
-1.00 \pm 0.08 \\
-0.95 \pm 0.09 \\
-0.92 \pm 0.12 \\
-1.01 \pm 0.18 \\
-0.95 \pm 0.06\end{array}$ \\
\hline \multicolumn{5}{|l|}{$44 \mathrm{GHz}$} \\
\hline $\begin{array}{l}\text { LFI24 } \ldots \ldots \ldots \\
\text { LFI25 } \ldots \ldots \ldots\end{array}$ & $\begin{array}{l}46.2 \pm 3.9 \\
24.9 \pm 3.7 \\
67.6 \pm 2.2\end{array}$ & $\begin{array}{r}100.9 \pm 8.3 \\
38.9 \pm 1.6 \\
58.9 \pm 4.4\end{array}$ & $\begin{array}{l}-0.83 \pm 0.04 \\
-0.91 \pm 0.01 \\
-0.95 \pm 0.01\end{array}$ & $\begin{array}{l}-0.73 \pm 0.03 \\
-1.16 \pm 0.01 \\
-0.79 \pm 0.02\end{array}$ \\
\hline \multicolumn{5}{|l|}{$30 \mathrm{GHz}$} \\
\hline $\begin{array}{l}\text { LFI27 } \\
\text { LFI28 }\end{array}$ & $\begin{array}{l}187.4 \pm 29.5 \\
122.2 \pm 10.2\end{array}$ & $\begin{array}{r}104.4 \pm 26.1 \\
40.7 \pm 7.2\end{array}$ & $\begin{array}{l}-0.87 \pm 0.05 \\
-0.88 \pm 0.05\end{array}$ & $\begin{array}{l}-0.82 \pm 0.14 \\
-0.91 \pm 0.13\end{array}$ \\
\hline
\end{tabular}

from a few regions in the Galactic plane where the temperature maps have large gradients over the pixel scale, causing the sky signal to leak into the noise map. In subsequent analysis steps we applied a $\sim 20 \%$ mask to remove these regions (see Fig. 20). Table 12 gives the standard deviation of normalised noise maps obtained for the three LFI frequencies. Deviations from unity trace the contribution of residual $1 / f$ noise in the final maps, which ranges from $0.2 \%$ at $70 \mathrm{GHz}$ to $4 \%$ at $30 \mathrm{GHz}$. A further Gaussianity test performed by calculating skewness and kurtosis of the normalised map after masking the galactic plane yielded null values within two standard deviations of 500 Gaussian simulations.

\subsection{Sensitivity}

Figure 21 compares the calibrated white noise sensitivity for the 22 LFI radiometers calculated from flight data and during ground tests performed both at instrument and satellite level. Error bars reported for values measured in flight and during satellite tests are statistical uncertainties derived from calculations performed on about 20 one-hour blocks of onground satellite data and one year of in-flight data. Error bars reported for values measured at instrument level, instead, represent the uncertainty between two different methods used to extrapolate the white noise sensitivity measured at test conditions ( $\sim 20 \mathrm{~K}$ input and $\sim 26 \mathrm{~K}$ front-end temperature) to flight conditions ( $\sim 2 \mathrm{~K}$ input and $\sim 20 \mathrm{~K}$ front-end temperature). Further details about this extrapolation are reported in Mennella et al. (2010).

Figure 21 shows general agreement in the sensitivity calculated in various test campaigns, with two outstanding exceptions, radiometers LFI24M and LFI21S, which show significantly improved noise levels in-flight. This resulted from an incorrect bias setting of these radiometers during ground tests (see Mennella et al. 2010), which was resolved during in-flight tuning.

In Fig. 21 values for LFI18M and LFI24M measured at instrument-level are not reported because these two radiometers failed. LFI18M was replaced with a spare unit and LFI24M was repaired before instrument delivery (see Mennella et al. 2010).
Table 13 summarizes the sensitivity numbers calculated during the first year of operations using methods and procedures outlined in Sect. 6.1 and described in detail in Zacchei et al. (2011), compared with scientific requirements. The measured sensitivity is in very good agreement with pre-launch expectations. While the white noise moderately exceeds the design specification, this performance is fully in line with the LFI science objectives.

The consistency of the noise estimates outlined in Table 10 was tested by comparing the jackknife noise maps, the white noise covariance matrices, and 101 noise Monte Carlo realisations (see Zacchei et al. 2011, for details). Noise covariance matrices and Monte Carlo simulations are dependent on estimated noise parameters, while the jackknife noise maps describe directly the noise in the final maps. Figure 22 shows pseudo- $C_{\ell}$ spectra obtained from the jackknife noise maps and Monte Carlo noise maps by anafast, compared to the high multipole tails $(1150<\ell<1800)$ predicted by white noise covariance matrices. In the analysis, we masked out a $20 \%$ region of the galactic plane and unsolved pixels (pixels that have HEALPix ${ }^{8}$ bad pixel value in noise maps), leaving us with sky fraction $f_{\text {sky }} \approx 0.8$.

Figure 23 compares the noise estimates obtained with the various methods. The green triangles refer to noise obtained from binned pure white noise Monte Carlo maps, i.e., maps containing no residual $1 / f$ noise. At all frequencies the high- $\ell$ mean of the jackknife noise map pseudo- $C_{\ell}$ spectrum weighted by the inverse sky coverage, $\left(f_{\text {sky }}\right)^{-1}$ (red), is in the $68 \%$ range of the noise Monte Carlo (black error bar). The point from the $30 \mathrm{GHz}$ jackknife noise map is almost $1-\sigma$ higher than the median value for the noise MC due to residual gradient leakage (galaxy, point sources etc.), which is strongest for the $30 \mathrm{GHz}$ channel. The noise power from the white noise covariance matrices and binned maps is always lower than the noise power from the full noise Monte Carlo and jackknife noise maps, as expected, due to residual $1 / f$ noise present even at high multipoles. This effect is especially pronounced in the $30 \mathrm{GHz}$ channels, which have larger knee frequencies (see Sect. 6.3).

${ }^{8}$ http://healpix.jpl.nasa.gov 
A\&A 536, A3 (2011)
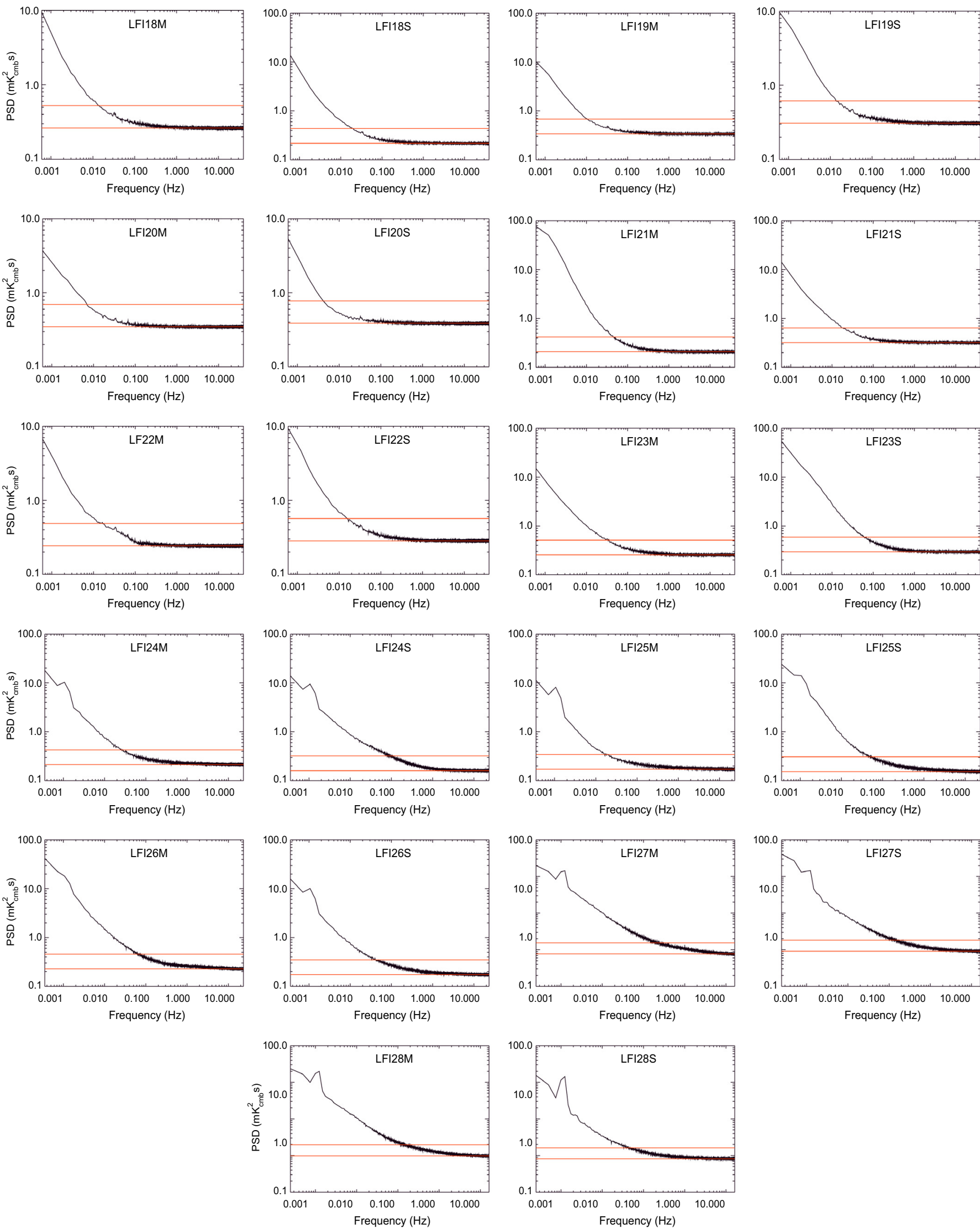

Fig. 18. Power spectral densities (PSDs) for the LFI radiometers. The lower red line marks the white noise level. The upper red line marks the level of equal contribution from white and $1 / f$ noise, so the intercept with the power spectrum marks the knee frequecy, $f_{\mathrm{knee}}$. 


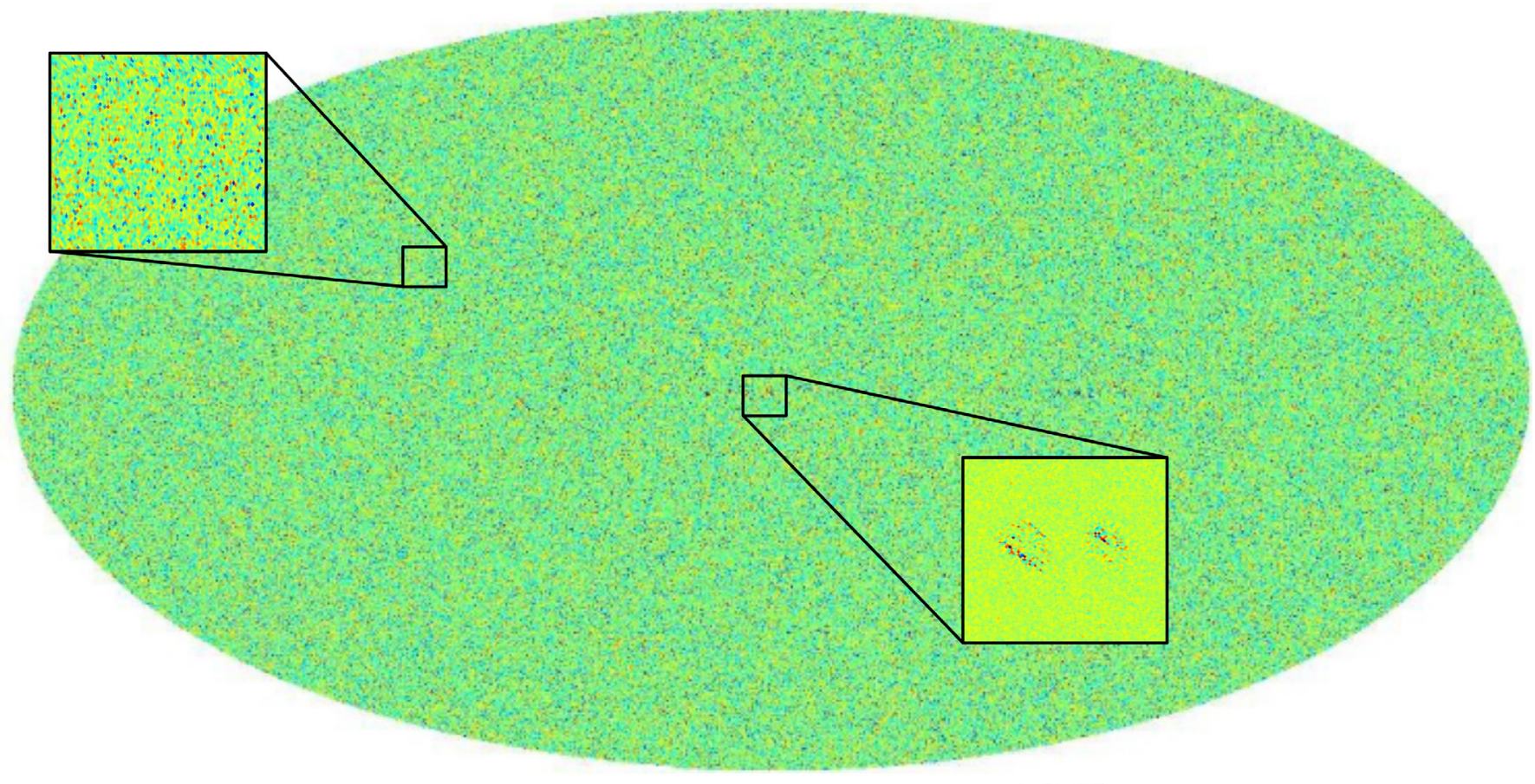

$-4.0$

4.0 Sigma

Fig. 19. $30 \mathrm{GHz}$ normalised jackknife noise map. We also highlight a region in the Galactic plane affected by leakage from strong foreground emission. The inset at high galactic latitude highlights the white nature of the noise.

Table 12. Standard deviation of normalised noise maps.

\begin{tabular}{lccc}
\hline \hline & $30 \mathrm{GHz}$ & $44 \mathrm{GHz}$ & $70 \mathrm{GHz}$ \\
\hline Standard deviation $\ldots . .$. & 1.039 & 1.016 & 1.002 \\
\hline
\end{tabular}

\subsection{1/f Noise properties}

Figure 24 compares the knee frequencies estimated from flight data with those measured in ground satellite calibration. Knee frequency and slope after one year of operations for all LFI radiometers were given earlier in Table 11 . With the exception of LFI23S, LFI24S, LFI27M, LFI28M, and LFI27S, for which the knee frequency measured in flight is higher than that measured on ground, the values are at or below requirements and are comparable within the error bars among different measurements.

The cause of the five high knee frequencies is still under investigation, but the impact on the scientific quality of the data is small. Destriping effectively removes correlated structures, limiting the impact of the high knee frequencies to a small $(<4 \%)$ increase of the noise variance (see Table 12 and Fig. 19).

As shown in the low- $\ell$ part of the angular power spectra in Fig. 22, the noise parameters estimated in flight are a good representation of the noise in the actual maps, and there is no need for a more complex noise model.

\section{First assessment of systematic effects}

The LFI design was driven by the need to suppress systematic effects well below instrument white noise. The differential receiver scheme, with reference loads cooled to $4 \mathrm{~K}$, greatly

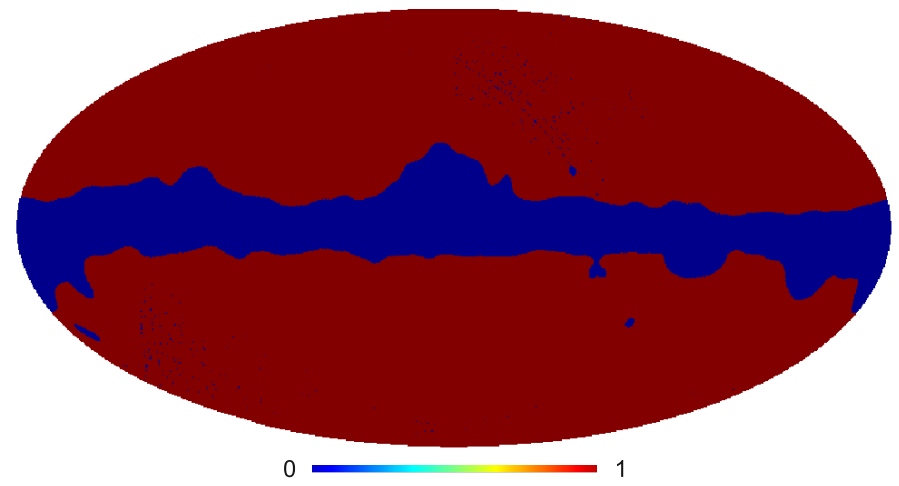

Fig. 20. $30 \mathrm{GHz}$ mask used to extract noise parameters from the corresponding noise map (masked points are shown in blue).

minimises the effect of $1 / f$ noise and common-mode fluctuations, such as thermal perturbations in the $20 \mathrm{~K}$ LFI focal plane. The use of a gain modulation factor (see Eq. (3)) largely compensates for spurious contributions from input offsets. Furthermore, diode averaging (Eq. (6)) allows us to cancel second-order correlations such as those originating from phase switch imbalances.

We have developed an error budget for systematic effects (Bersanelli et al. 2010) as a reference for both instrument design and data analysis. Our goal is to ensure that each systematic effect is rejected to the specified level, either by design or by robust removal in software. At this stage, the following effects are relevant:

- 1/f noise (Sect. 6.3);

- $1 \mathrm{~Hz}$ frequency spikes; 

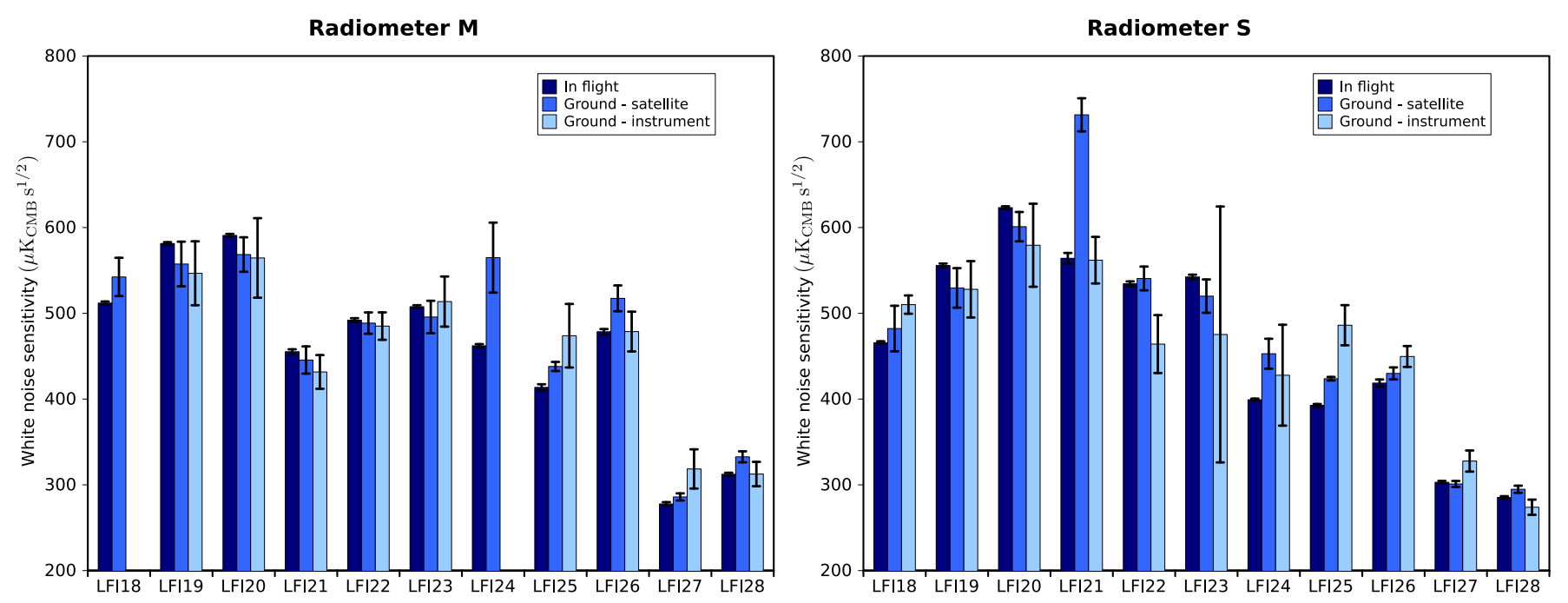

Fig. 21. Comparison of white noise sensitivity values calculated in flight and on ground during the satellite-level and instrument-level test campaigns. Values for LFI18M and LFI24M measured at instrument-level are not reported because these two radiometers failed before instrument delivery and were replaced and repaired, respectively.

Table 13. White noise sensitivity of the LFI frequency channels compared with requirements.

\begin{tabular}{ccc}
\hline \hline Channel & $\begin{array}{c}\text { Measurement } \\
{\left[\mu \mathrm{K}_{\mathrm{CMB}} \mathrm{s}^{1 / 2}\right]}\end{array}$ & $\begin{array}{c}\text { Requirement } \\
{\left[\mu \mathrm{K}_{\mathrm{CMB}} \mathrm{s}^{1 / 2}\right]}\end{array}$ \\
\hline $70 \mathrm{GHz} \ldots \ldots \ldots \ldots \ldots$ & 152.6 & 119 \\
$44 \mathrm{GHz} \ldots \ldots \ldots \ldots \ldots$ & 173.1 & 119 \\
$30 \mathrm{GHz} \ldots \ldots \ldots \ldots \ldots$ & 146.8 & 119 \\
\hline
\end{tabular}

- thermal fluctuations in the back-end modules driven by temperature oscillations from the transponder during the first survey;

- thermal fluctuations in the $20 \mathrm{~K}$ focal plane;

- thermal fluctuations of the $4 \mathrm{~K}$ reference loads.

For each of these effects we used flight data and information from ground tests to build timelines, maps, and angular power spectra that represent our best knowledge of their impact on the scientific analysis. Figure 25 shows maps and histograms of the combination of all the above mentioned systematic effects for the three LFI channels. The figure shows that the 30 and $44 \mathrm{GHz}$ channels display a residual level of spurious signals about twice larger than the $70 \mathrm{GHz}$ channel. As shown in the following sections, this is caused by temperature fluctuations of $4 \mathrm{~K}$ reference loads that are significantly larger at 30 and $44 \mathrm{GHz}$ than at $70 \mathrm{GHz}$.

A convenient way to assess the effect on angular power spectra is to calculate the ratio $\rho_{\ell}=C_{\ell}^{\text {syst }} / C_{\ell}^{\text {noise }}$, where $C_{\ell}^{\text {syst }}$ is the angular power spectrum of the systematic effect map and $C_{\ell}^{\text {noise }}$ is the angular power spectrum of the instrument noise contribution including the residual $1 / f$ component remaining after destriping. Figure 26 shows $\rho_{\ell}$ for the three LFI frequency channels. In each panel we have plotted the spectrum obtained from the global maps in Fig. 25 and the spectra derived from the singlecomponent maps (see next sections).

Figure 26 shows that $\rho_{\ell}$ is in the range $10^{-1}-10^{-2}$ for $\ell$ in the range $2-20$, and $\rho_{\ell}<10^{-2}$ for $\ell>10$. The figure also shows that at 30 and $44 \mathrm{GHz}$ the largest contribution to systematic effects comes from temperature fluctuations of the $4 \mathrm{~K}$ reference loads, while the residual systmatic uncertainty in the $70 \mathrm{GHz}$ channel is mainly caused by back-end temperature fluctuations and, at small angular scales, by frequency spikes. Table 14 gives an overview of our current assessment of residual peak-to-peak and rms systematic effects per pixel on LFI temperature maps. Maps were made with $N_{\text {side }}=512$ at $30 \mathrm{GHz}$ and $N_{\text {side }}=1024$ at 44 and $70 \mathrm{GHz}$. Corresponding pixel sizes are $\sim 6.8$ and $\sim 3.4$.

Further advances in the data analysis pipeline will be aimed at removing spikes from the $70 \mathrm{GHz}$ data, improving relative calibration to account for thermally driven fluctuations, and further suppressing spurious fluctuations caused by $4 \mathrm{~K}$ temperature instabilities at 30 and $44 \mathrm{GHz}$.

\subsection{Frequency spikes}

Spikes are seen in the radiometer outputs in the frequency domain at multiples of $1 \mathrm{~Hz}$. These "frequency spikes" were first detected during ground tests, and are caused by pickup from the clock of the housekeeping electronics (Meinhold et al. 2009; Mennella et al. 2010). The pickup occurs between the detector diodes and the DAE gain stage. Frequency spikes are present at some level in the output from all detectors, but affect the $44 \mathrm{GHz}$ data most strongly because of the low voltage output and high DAE gain values in that channel. In this section we provide estimates of the residual systematic effect after frequency spike removal at $44 \mathrm{GHz}$, and the effect caused by the spikes without any removal at 30 and $70 \mathrm{GHz}$.

In the time domain, the frequency domain spikes comprise a one second square wave with a rising edge near $0.5 \mathrm{~s}$ and a falling edge near $0.75 \mathrm{~s}$ in on-board time. During the first year of operations we did not observe any deviation in either the phase or the shape of these signals.

Templates of these spurious square waves obtained from the output voltages have been used to remove this effect from the data before differentiation (procedures and algorithms are described in Zacchei et al. 2011). Although we could in principle 


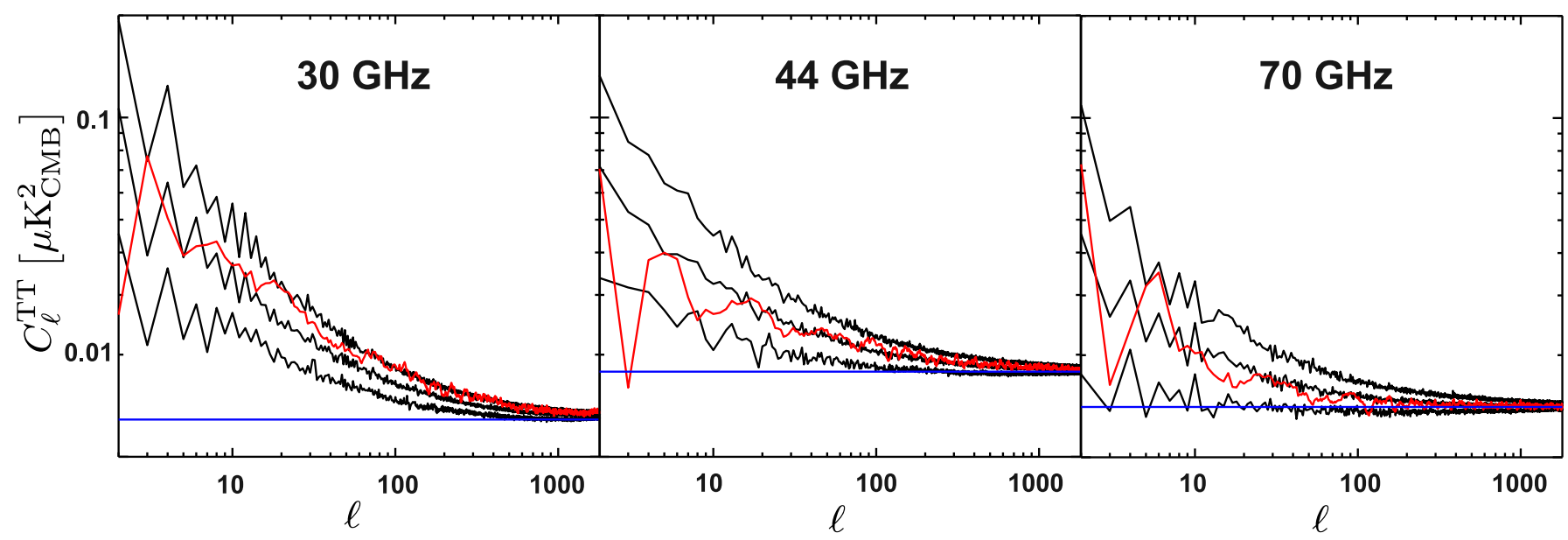

Fig. 22. The noise angular power spectra. Black: noise Monte Carlo (median, $16 \%$ quantile, and $84 \%$ quantile). Red: jackknife noise map pseudo$C_{\ell}$. Blue: values from white noise covariance matrices.

Table 14. Effect on maps of the main systematic effects, in $\mu \mathrm{K}_{\mathrm{CMB}} \mathrm{s}^{1 / 2}$.

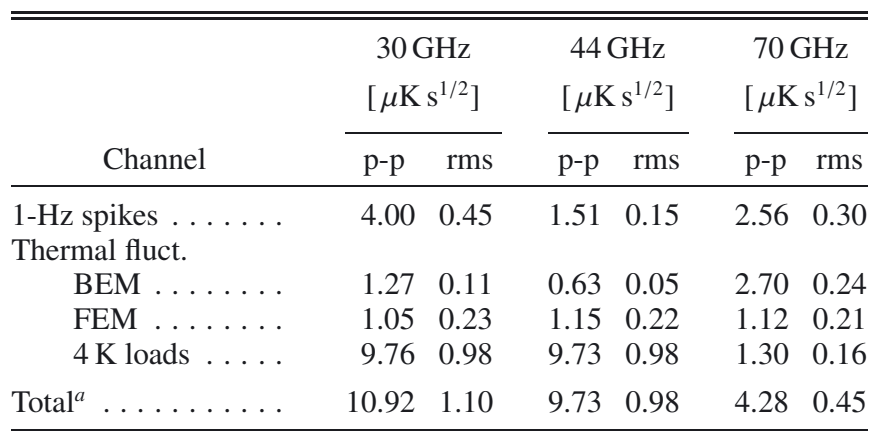

Notes. (a) Estimated from maps combining all systematic effects (Fig. 25).

apply the removal process to all data, we decided to remove the spikes only from the $44 \mathrm{GHz}$ data, which are the most affected by this effect.

To estimate the residual in the spike removal process caused by slow variations in the square wave amplitude, we used a simple $\chi^{2}$ minimisation procedure to estimate the amplitude of the template for each hour of data, then smoothed the amplitude by simple binning with a 10-day window size. We took this smoothed amplitude as our estimate of the true time variation of the spike signal, and calculated the residual error that would result if we approximated this time-varying signal with a constant template in the spike removal process. Although we could use, in principle, the time-varying template to remove the effect, we decided to use the simplest and most robust approach of a constant-amplitude template. The possibility to implement a model that accounts for slow spike amplitude drifts will be considered for future data releases.

Templates were also used to generate maps for each frequency channel to determine the impact of the spike signal on the science results. These maps and the corresponding histograms are shown in Fig. 27. For the $44 \mathrm{GHz}$ channel we also show the residual effect after removing the constant template, showing that after removal the residual effect is reduced by a factor of about 20 .

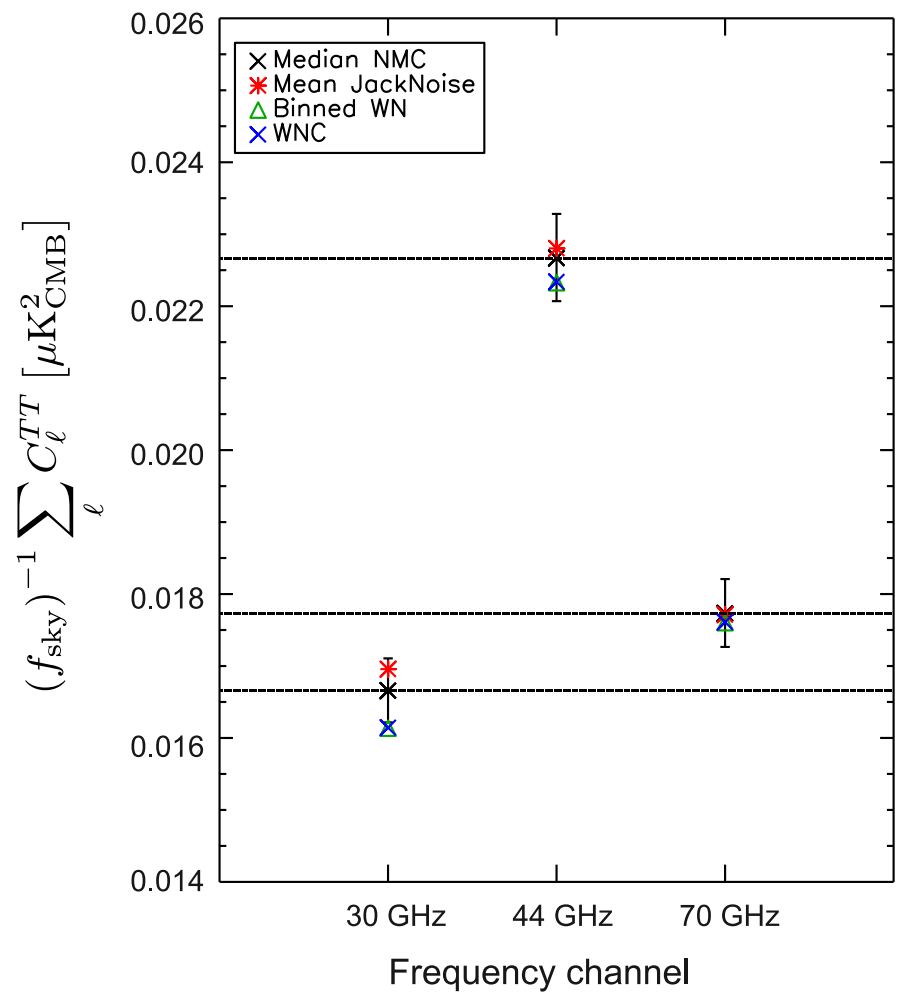

Fig. 23. Comparison of noise estimates from different methods. Black: noise from high- $\ell$ means of noise Monte Carlo statistics. Red: noise from the high- $\ell$ mean of the jackknife noise map pseudo- $C_{\ell}$ spectra. Green: noise from the binned white noise Monte Carlo maps. Blue: noise from white noise covariance matrices. Error bars are relative to values from Monte Carlo simulations. We have weighted all the values with the inverse of the analysed sky fraction, $f_{\text {sky }}^{-1}$, to represent the noise levels of temperature $C_{\ell}$ spectra that would result from using full sky maps.

\subsection{Thermal fluctuations}

The LFI is sensitive to temperature fluctuations of the warm back-end unit, the $20 \mathrm{~K}$ focal plane, and the $4 \mathrm{~K}$ reference loads. In the first two, temperature variations impact the sky and reference load signals at a similar level, so that in the differential 
Radiometer M

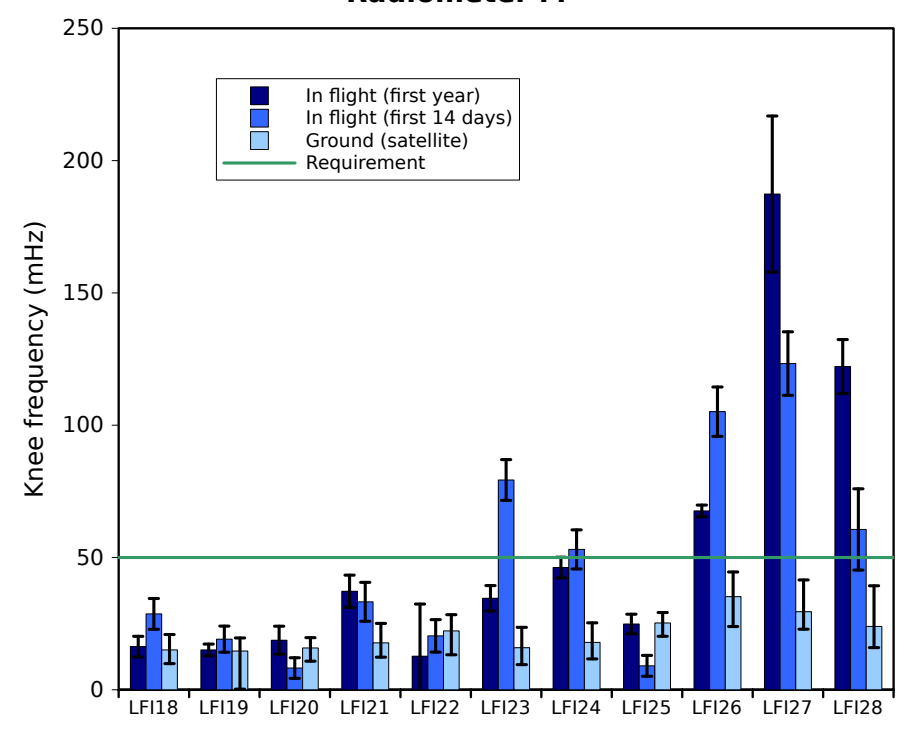

Radiometer S

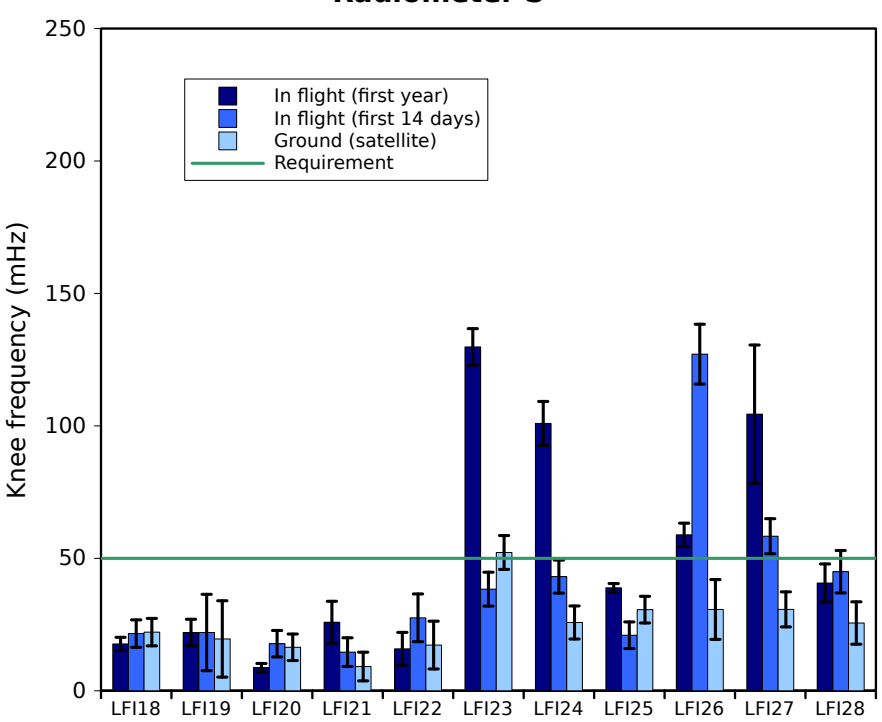

Fig. 24. Comparison of $1 / f$ knee frequencies measured in flight and on ground during the satellite-level test campaign.

radiometric output the residual spurious variations are reduced by more than one order of magnitude. Fluctuations at the level of the $4 \mathrm{~K}$ reference loads, instead, transfer directly to the radiometric output, and may represent a more critical source of systematic errors.

The effects of low frequency thermal fluctuations are strongly suppressed by the spacecraft spin itself and the destriping map-making codes (Zacchei et al. 2011). High frequency thermal fluctuations are strongly damped by the thermomechanical structure of the spacecraft and instruments (Tomasi et al. 2009). The dominant effects, then, come from fluctuations at frequencies in a range around the spin frequency.

We estimate the impact on LFI science of thermal fluctuations as follows:

- start from a time ordered datastream of a temperature sensor representative of the temperature behaviour of the considered thermal stage;

- low-pass filter to remove high-frequency sensor noise;

- apply thermal transfer functions where appropriate to obtain the physical temperature behaviour at the level of the receiver components sensitive to the fluctuation;

- apply the radiometric transfer function to convert the physical temperature fluctuation into antenna temperature fluctuation;

- resample at the scientific sampling frequency, calibrate, and build differenced time-ordered data;

- build maps with Madam.

These maps have been generated combining actual flight housekeeping data with thermal and radiometric transfer functions obtained from flight and ground-test data (Terenzi et al. 2009). They represent, therefore, our current best estimate of the impact of the individual effects on the science in flight conditions.

As explained in Sect. 5.3, in the future thermal fluctuations from the back-end unit will be included in the calibration model. This will reduce the need to remove the spurious signal from the time ordered data during map-making.

\subsubsection{Back-end temperature fluctuations}

As mentioned in Sect. 5.1, for the first 258 days of the mission the satellite transponder was switched on only for the daily telecommunication period. This induced quasi-sinusoidal fluctuations in the Planck service module temperature that propagated to the LFI back-end unit, and were recorded by the instrument housekeeping sensors at the level of $\pm 0.2 \mathrm{~K} \mathrm{day}^{-1}$.

These temperature fluctuations drive variations in the total power voltage output of both sky and reference detectors, which largely disappear in the difference, shown in Fig. 13. The effect on the undifferenced total power data, however, was useful in calculating correlation coefficients between temperature and voltage outputs. These coefficients have been used to estimate the residual effect on time-ordered data and maps. Figure 28 shows this correlation for the detector LFI27M-00.

Figure 29 shows the expected systematic effects of temperature fluctuations of the back-end unit during the first year of operations. The peak-to-peak effect is $\sim 1 \mu \mathrm{K}_{\mathrm{CMB}}$, and the rms is well below $1 \mu \mathrm{K}_{\mathrm{CMB}}$.

\subsubsection{Front-end temperature fluctuations}

Another source of temperature fluctuations is the Planck sorption cooler (Morgante et al. 2009; Planck Collaboration 2011b), which has temperature variations of the order of $\sim 0.5 \mathrm{~K}$ peak-topeak at the cold end attached to the LFI focal plane unit. These fluctuations are damped by a thermal stabilisation assembly (TSA; Planck Collaboration 2011b) down to about $100 \mathrm{mK}$ peak-to-peak at low frequency.

During the second half of the first year of operations the expected cooler degradation led to an increase of the cold end temperature and the low-frequency fluctuations. The temperature of the radiometers increased by about $0.2 \mathrm{~K}$, with fluctuations running about $30 \mathrm{mK}$ peak-to-peak. We estimated the effect of these fluctuations on the science data using ground-measured transfer functions that include the effect of temperature on both gain and noise. Figure 30 shows the calculated effects for the three 
A. Mennella et al.: Planck early results. III.

\section{$30 \mathrm{GHz}$}
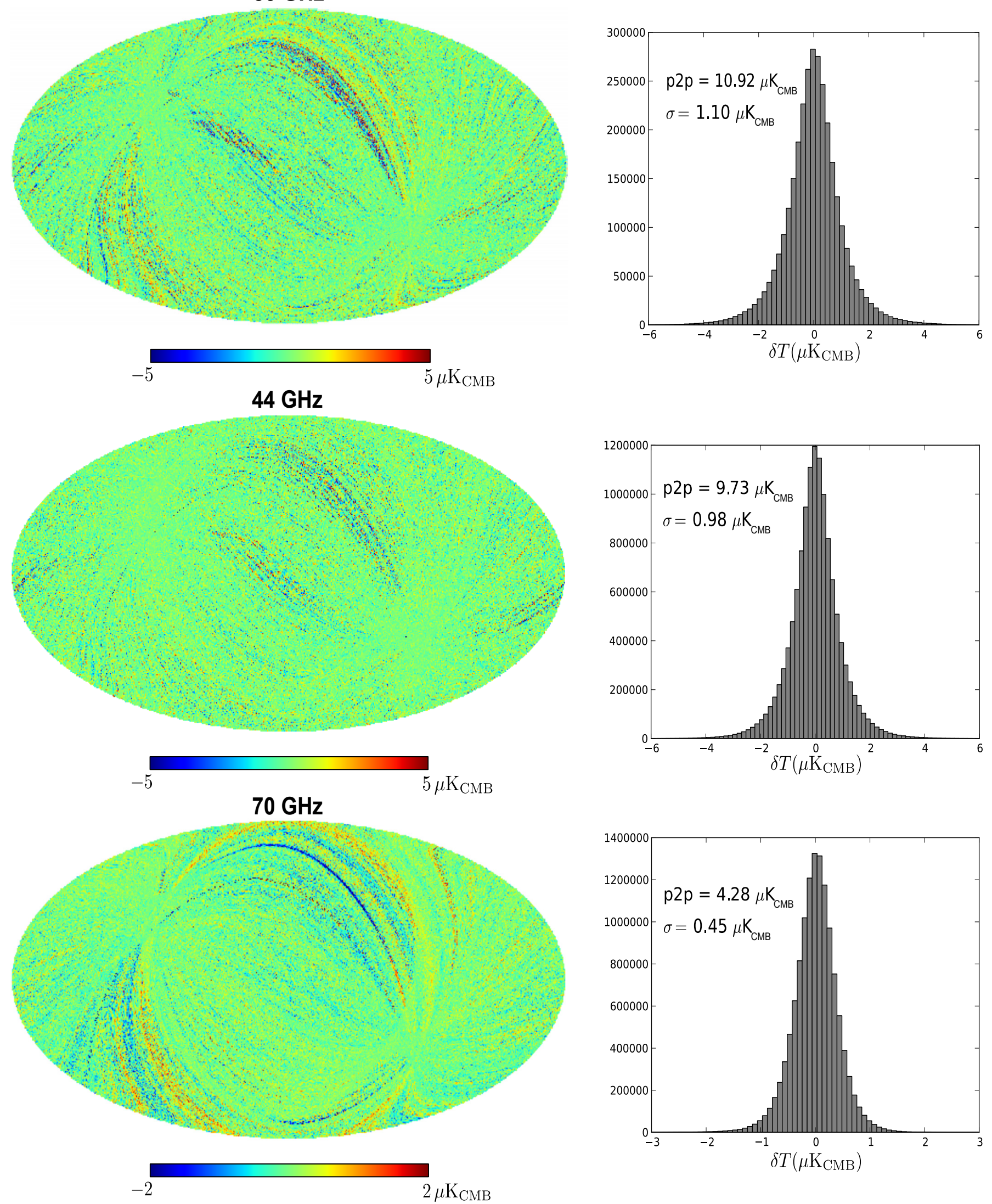

Fig. 25. Maps of the combined systematic effects from frequency spikes and thermal fluctuations. 

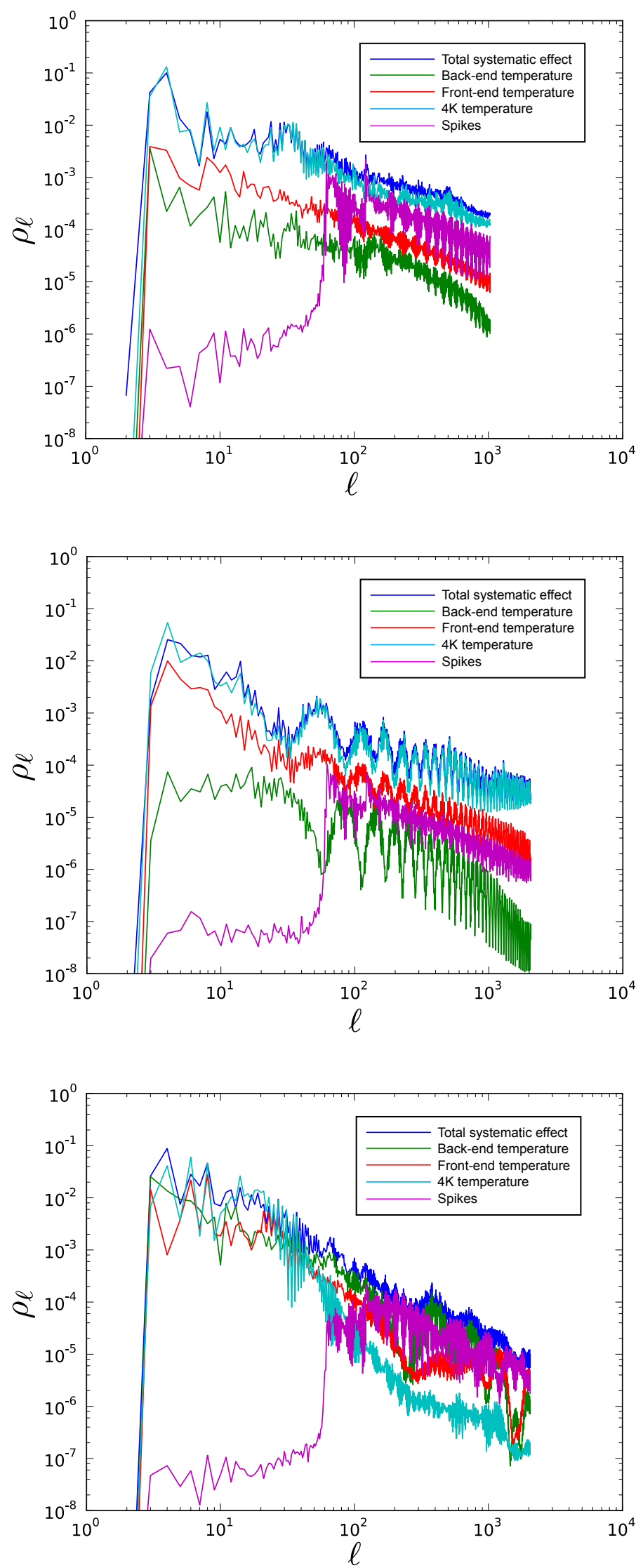

Fig. 26. Ratio $\rho_{\ell}=C_{\ell}^{\text {syst }} / C_{\ell}^{\text {noise }}$ for the three LFI frequency channels (top: $30 \mathrm{GHz}$; middle: $44 \mathrm{GHz}$; bottom: $70 \mathrm{GHz}$ ). In each panel we have plotted both the global spectrum obtained from the maps in Fig. 25 and the individual spectra calculated from the single-component maps.
LFI frequency channels. As in the case of back-end temperature fluctuations, the overall effect is $\sim 1 \mu \mathrm{K}_{\mathrm{CMB}}$ peak-to-peak and $0.2 \mu \mathrm{K}_{\mathrm{CMB}} \mathrm{rms}$.

\subsection{3. $4 \mathrm{~K}$ reference load temperature fluctuations}

The temperature stability of the $4 \mathrm{~K}$ reference loads, attached to the HFI $4 \mathrm{~K}$ mechanical box, is a key factor in the LFI systematic effects budget. Temperature fluctuations of the loads are driven primarily by fluctuations at the $20 \mathrm{~K}$ cold-end interface, which propagate to the LFI reference loads by conduction through the HFI mounting struts and radiation from the LFI. See Planck Collaboration (2011b) for details.

The $70 \mathrm{GHz}$ reference loads are mounted close to the HFI $4 \mathrm{~K}$ plate and are stabilised by proportional-integral-derivative (PID) active controllers that remove almost completely low frequency fluctuations. The loads at 30 and $44 \mathrm{GHz}$ are farther from the HFI $4 \mathrm{~K}$ plate and closer to the HFI mounting struts; their temperature is less stable. Details of temperature sensor measurements are provided in Planck Collaboration (2011b).

This difference is reflected in the maps shown in Fig. 31, where the residual systematic effect in the 30 and $44 \mathrm{GHz}$ channels is $\sim 10$ times larger than in the $70 \mathrm{GHz}$ channel. Nevertheless, given the reduction provided by destriping, the residual systematic is several times below the instrument noise, as shown by the angular power spectra in Fig. 26. Further work will be aimed at correlating radiometric and housekeeping data to further reduce the residual effect caused by these fluctuations.

\section{Summary of main performance parameters}

Table 15 gives a top-level summary of instrument performance parameters measured in flight. Optical properties have been successfully reconstructed using Jupiter transits and the main parameters are in agreement with pre-launch estimates. The white noise sensitivity agrees with ground measurements; for some channels sensitivity improved after in-flight bias tuning. Parameters describing the $1 / f$ noise component are in line with ground measurements and the $50 \mathrm{mHz}$ requirement except at $30 \mathrm{GHz}$. That channel has a knee frequency over $100 \mathrm{mHz}$, which is, however, well-handled by destriping. Absolute photometric calibration based on the CMB dipole yields an overall statistical uncertainty of $\lesssim 1 \%$. Variations due to slow instrumental variations are traced by the calibration pipeline, yielding an overall uncertainty ranging between $0.05 \%$ and $0.1 \%$. The residual systematic uncertainty is of the order of $1 \mu \mathrm{K}$ rms per pixel. Average colour corrections are provided in Table 2.

\section{Conclusions}

Since the start of nominal operations, the LFI has shown excellent stability in all measured parameters. The instrument uninterruptedly observed the microwave sky with negligible data loss and less than $1 \%$ discarded data because of anomalies. Typical variations in the instrumental output were less than $1 \%$ on time scales of several days and were mainly driven by slow thermal fluctuations.

The main beams have been reconstructed down to $-20 \mathrm{~dB}$ using Jupiter as a source, with results closely matching those expected from simulations. In-flight measurements of white 
A. Mennella et al.: Planck early results. III.
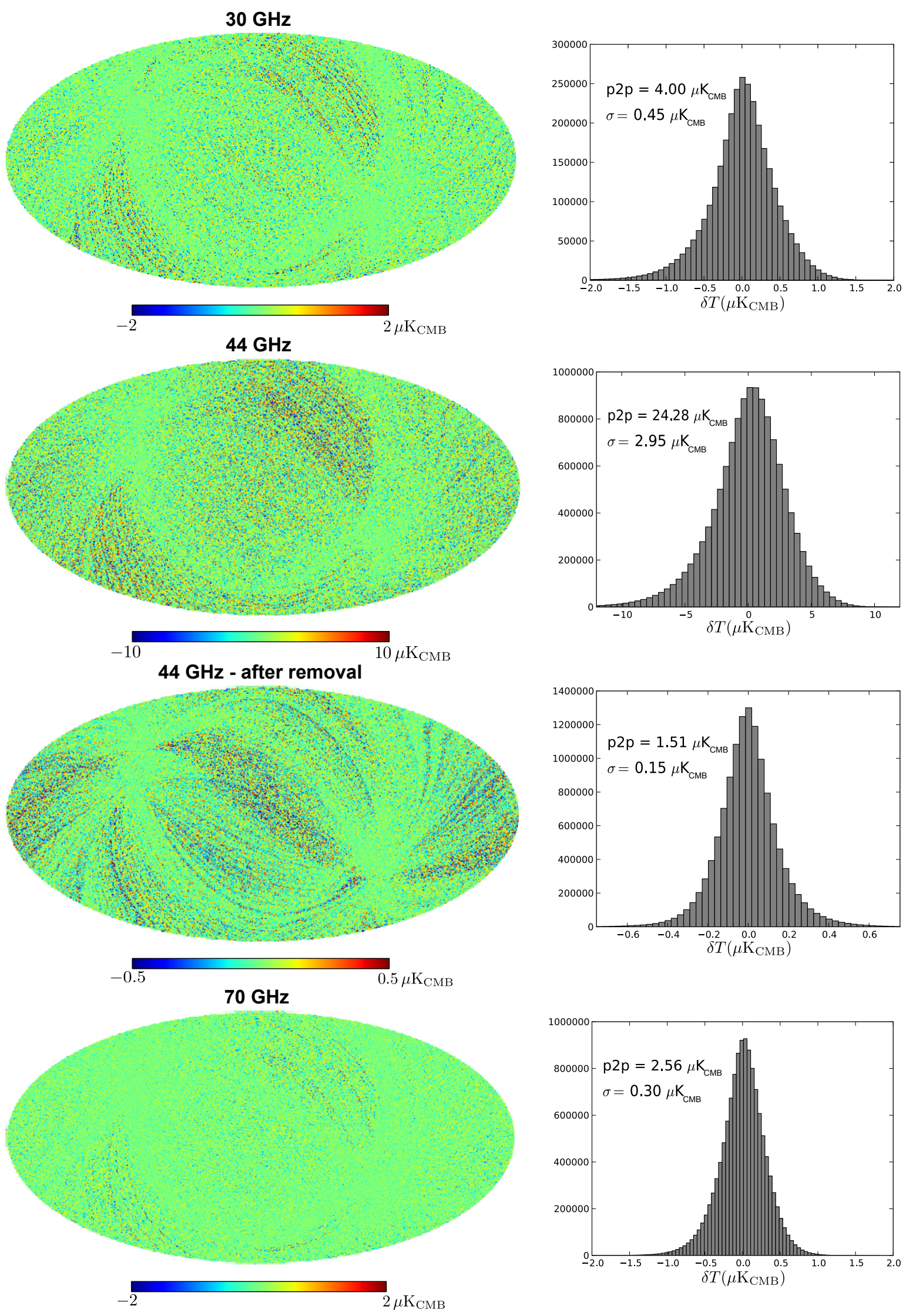

Fig. 27. Simulated spike maps for each channel, and the residual after removal for the $44 \mathrm{GHz}$ channel. Maps were made using constant-amplitude templates to generate spike TODs. The data used to generate the residual map were obtained by subtracting a spike TOD with time-varying amplitude from a constant-amplitude spike TOD. 
Table 15. Summary of main LFI performance parameters.

\begin{tabular}{|c|c|c|c|}
\hline Parameter & $30 \mathrm{GHz}$ & $44 \mathrm{GHz}$ & $70 \mathrm{GHz}$ \\
\hline Centre frequency $[\mathrm{GHz}] \ldots \ldots \ldots \ldots \ldots \ldots$ & 28.5 & 44.1 & 70.3 \\
\hline FWHM $\left[{ }^{\prime}\right] \ldots \ldots \ldots \ldots \ldots \ldots \ldots \ldots \ldots$ & 32.65 & 27.92 & 13.01 \\
\hline Ellipticity & 1.38 & 1.28 & 1.26 \\
\hline White noise sensitivity $\left[\mu \mathrm{K}_{\mathrm{CMB}} \mathrm{s}^{1 / 2}\right]$ & 146.8 & 173.1 & 152.6 \\
\hline$f_{\text {knee }}[\mathrm{mHz}] \ldots \ldots \ldots \ldots \ldots$ & 113.7 & 56.2 & 29.5 \\
\hline $1 / f$ slope..$\ldots \ldots \ldots \ldots \ldots \ldots \ldots \ldots \ldots$ & -0.87 & -0.89 & -1.03 \\
\hline Absolute calibration uncertainty $[\%] \ldots \ldots \ldots \ldots$ & 1 & 1 & 1 \\
\hline Relative calibration uncertainty $[\%] \ldots \ldots \ldots \ldots$ & 0.05 & 0.07 & 0.12 \\
\hline Systematic effects uncertainty $\left[\mu \mathrm{K}_{\mathrm{CMB}}\right] \ldots \ldots \ldots$ & 1.10 & 0.98 & 0.45 \\
\hline
\end{tabular}

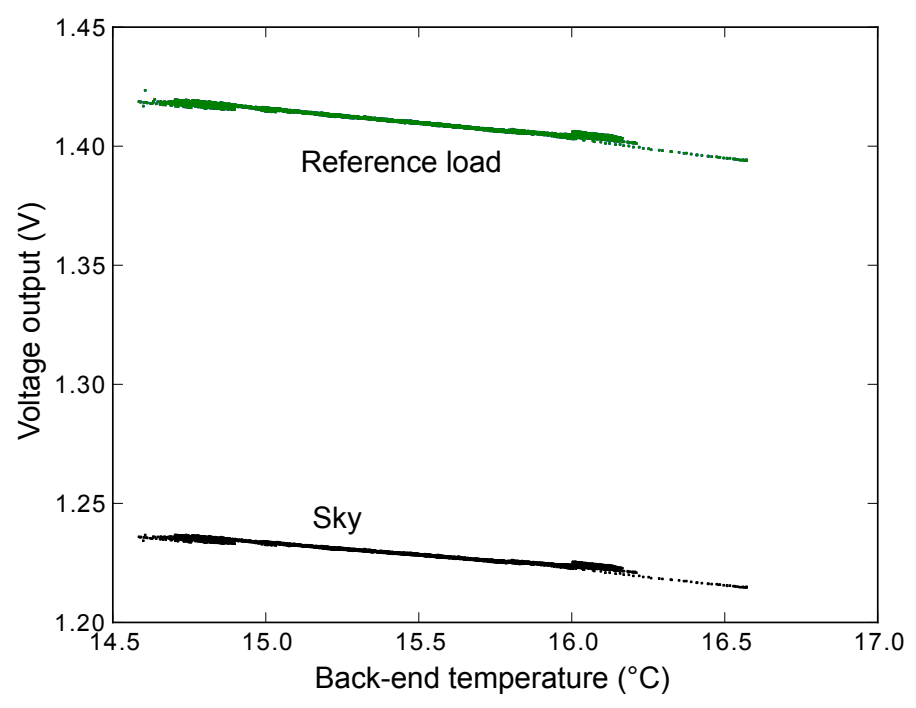

Fig. 28. Correlation between back-end temperature and total power voltage output relative to the LFI detector LFI27M-00.

noise sensitivity are in good agreement with ground results, with significant improvements in some channels thanks to inflight bias tuning. The impact of low frequency noise is small, especially at $70 \mathrm{GHz}$ where the measured knee frequency is about $30 \mathrm{mHz}$, almost a factor of two below the requirement. At $30 \mathrm{GHz}$ the $1 / f$ noise is higher than measured on the ground, with a knee frequency of about $100 \mathrm{mHz}$. Residual fluctuations in the timestreams, however, are effectively removed during map-making, as expected from several pre-launch simulation studies (Kurki-Suonio et al. 2009; Poutanen et al. 2004; Keihänen et al. 2004). We have shown that the noise increase due to the $1 / f$ component is a few percent at 30 and $44 \mathrm{GHz}$, and only $0.2 \%$ at $70 \mathrm{GHz}$.

Photometric calibration is based on the CMB dipole via an iterative procedure explained in Zacchei et al. (2011). Excellent absolute calibration accuracy of $\lesssim 1 \%$ will be improved still further in future analyses by using the orbital dipole as an absolute calibrator. Our current relative calibration traces gain variations on timescales larger than 5-10 days, yielding an overall statistical accuracy in the range $0.05-0.1 \%$. Thermally-driven gain fluctuations on smaller timescales are currently not implemented in our gain model and contribute as a systematic uncertainty in the final maps. A new version of the gain model, now being developed, will take into account the effect of such fluctuations to further reduce this residual uncertainty.

We have presented a preliminary analysis of all the systematic effects that are relevant at this stage of the analysis. Their impact on LFI science has been evaluated by projecting each effect on full-sky maps and angular power spectra. The combined residual effect from frequency spikes and thermal instabilities (in the $4 \mathrm{~K}, 20 \mathrm{~K}$, and $300 \mathrm{~K}$ stages) is several order of magnitudes below the instrumental noise at all angular scales smaller than $10^{\circ}$, and less than $10 \%$ at larger scales.

The overall performance of LFI as measured in-flight demonstrates an instrument with an unprecedented combination of sensitivity, angular resolution, and suppression of systematic errors for full-sky imaging at these frequencies. Our preliminary assessment shows that even without dedicated decorrelation of thermal effects, the LFI is already largely immune to instrumental effects, with prospects of further suppression after implementing a more representative gain model and temperature fluctuation removal algorithms.

One more year of continuous observations is currently planned for Planck, with a further LFI extension to the maximum lifetime allowed by the sorption cooler. Everything to date suggests that the instrument will maintain its performance throughout the remaining period and provide rich and high-quality scientific data that will be explored for many years to come.

Acknowledgements. Planck is too large a project to allow full acknowledgement of all contributions by individuals, institutions, industries, and funding agencies. The main entities involved in the mission operations are as follows. The European Space Agency operates the satellite via its Mission Operations Centre located at ESOC (Darmstadt, Germany) and coordinates scientific operations via the Planck Science Office located at ESAC (Madrid, Spain). Two Consortia, comprising around 50 scientific institutes within Europe, the USA, and Canada, and funded by agencies from the participating countries, developed the scientific instruments LFI and HFI, and continue to operate them via Instrument Operations Teams located in Trieste (Italy) and Orsay (France). The Consortia are also responsible for scientific processing of the acquired data. The Consortia are led by the Principal Investigators: J.-L. Puget in France for HFI (funded principally by CNES and CNRS/INSU-IN2P3) and N. Mandolesi in Italy for LFI (funded principally via ASI). NASA's US Planck Project, based at JPL and involving scientists at many US institutions, contributes significantly to the efforts of these two Consortia. In Finland, the Planck LFI $70 \mathrm{GHz}$ work was supported by the Finnish Funding Agency for Technology and Innovation (Tekes). This work was also supported by the Academy of Finland, CSC, and DEISA (EU).

Some of the results in this paper have been derived using the HEALPix package (Górski et al. 2005).

A description of the Planck Collaboration and a list of its members can be found at http: //www.rssd.esa.int/index.php?project=PLANCK\& page=Planck_Collaboration. 
A. Mennella et al.: Planck early results. III.

\section{$30 \mathrm{GHz}$}
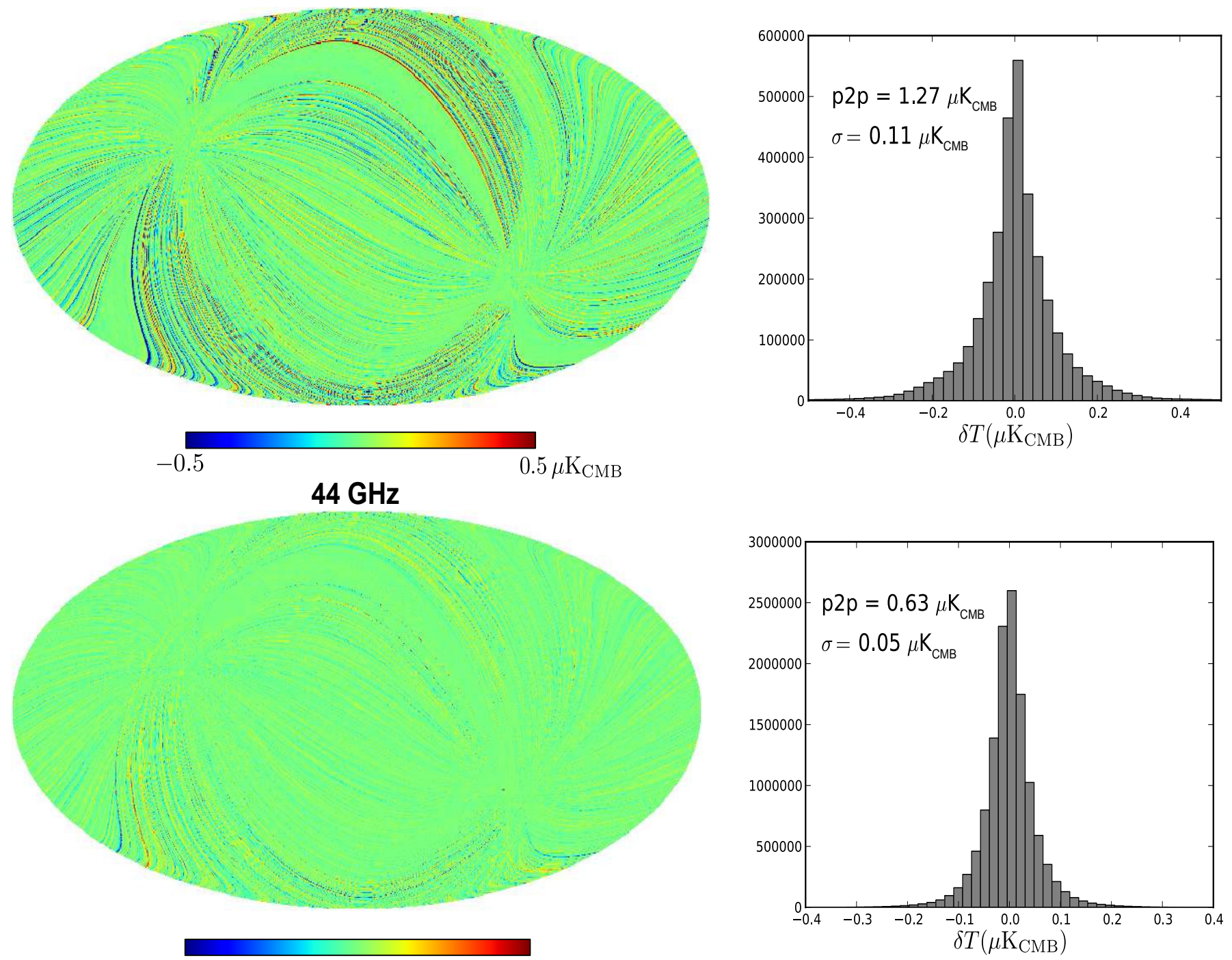

$-0.5$ $0.5 \mu \mathrm{K}_{\mathrm{CMB}}$

$70 \mathrm{GHz}$

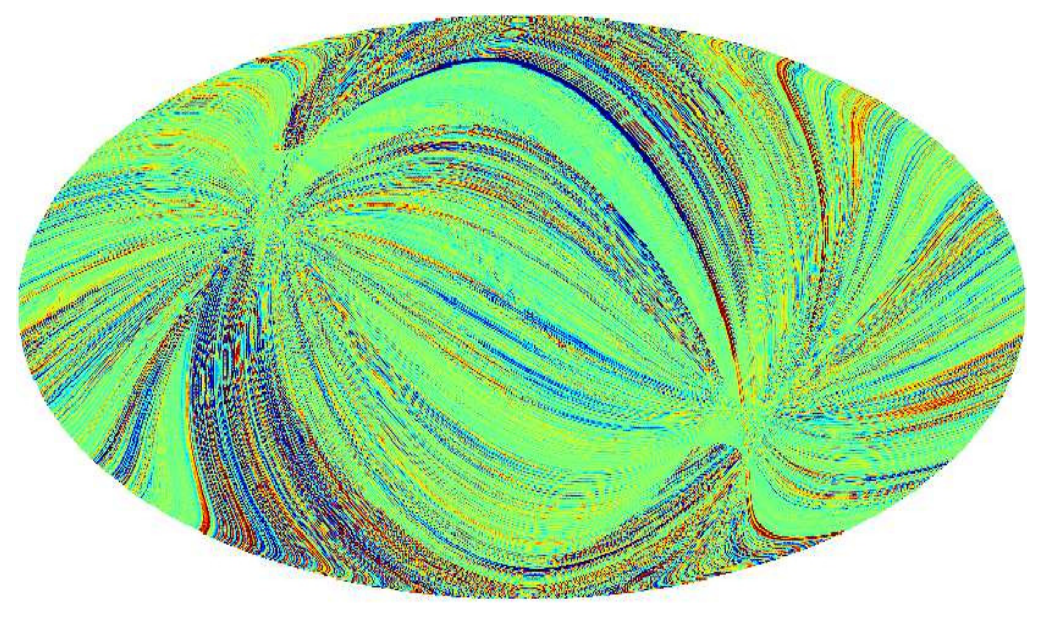

$$
-0.5
$$

\section{$0.5 \mu \mathrm{K}_{\mathrm{CMB}}$}
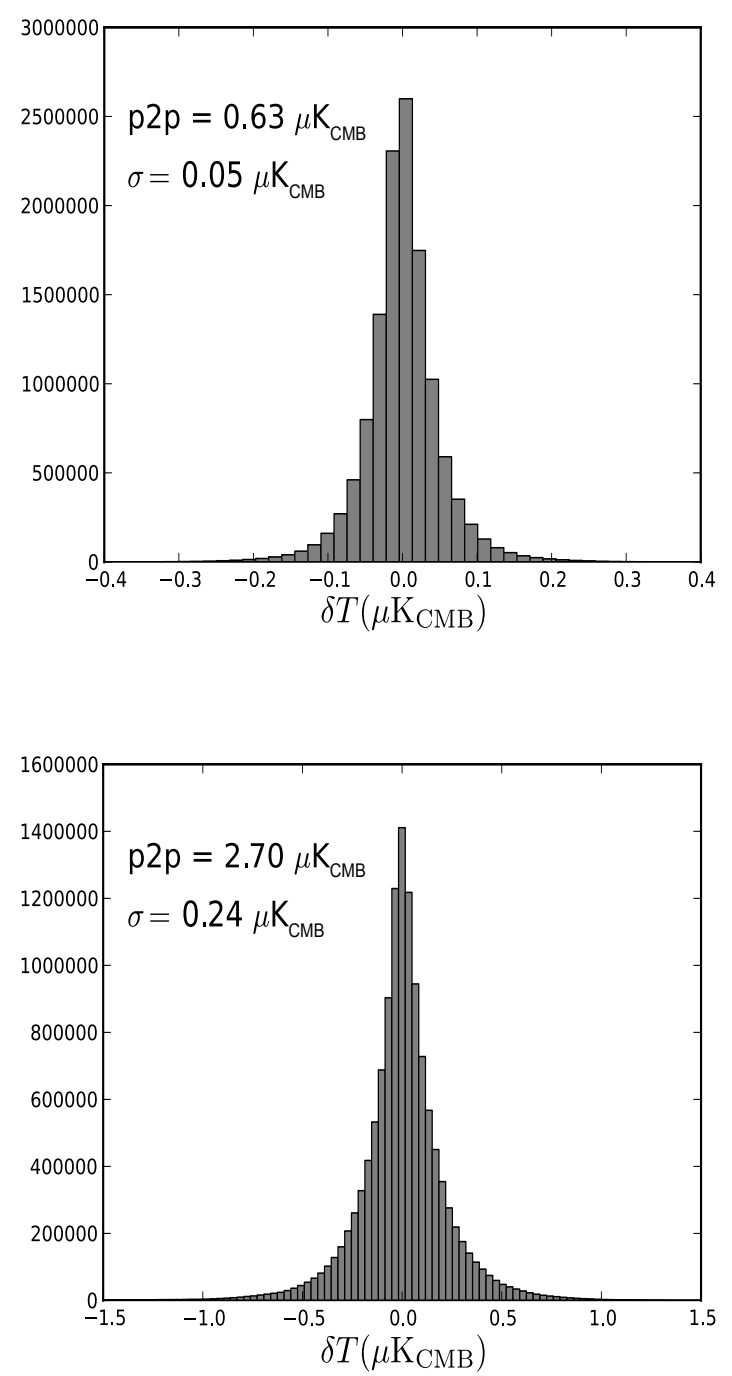

Fig. 29. Simulation of the systematic effects of temperature fluctuations of the LFI warm back-end unit. Maps are $N_{\text {side }}=512$ at $30 \mathrm{GHz}$, and $N_{\text {side }}=1024$ at 44 and $70 \mathrm{GHz}$. 


\section{$30 \mathrm{GHz}$}
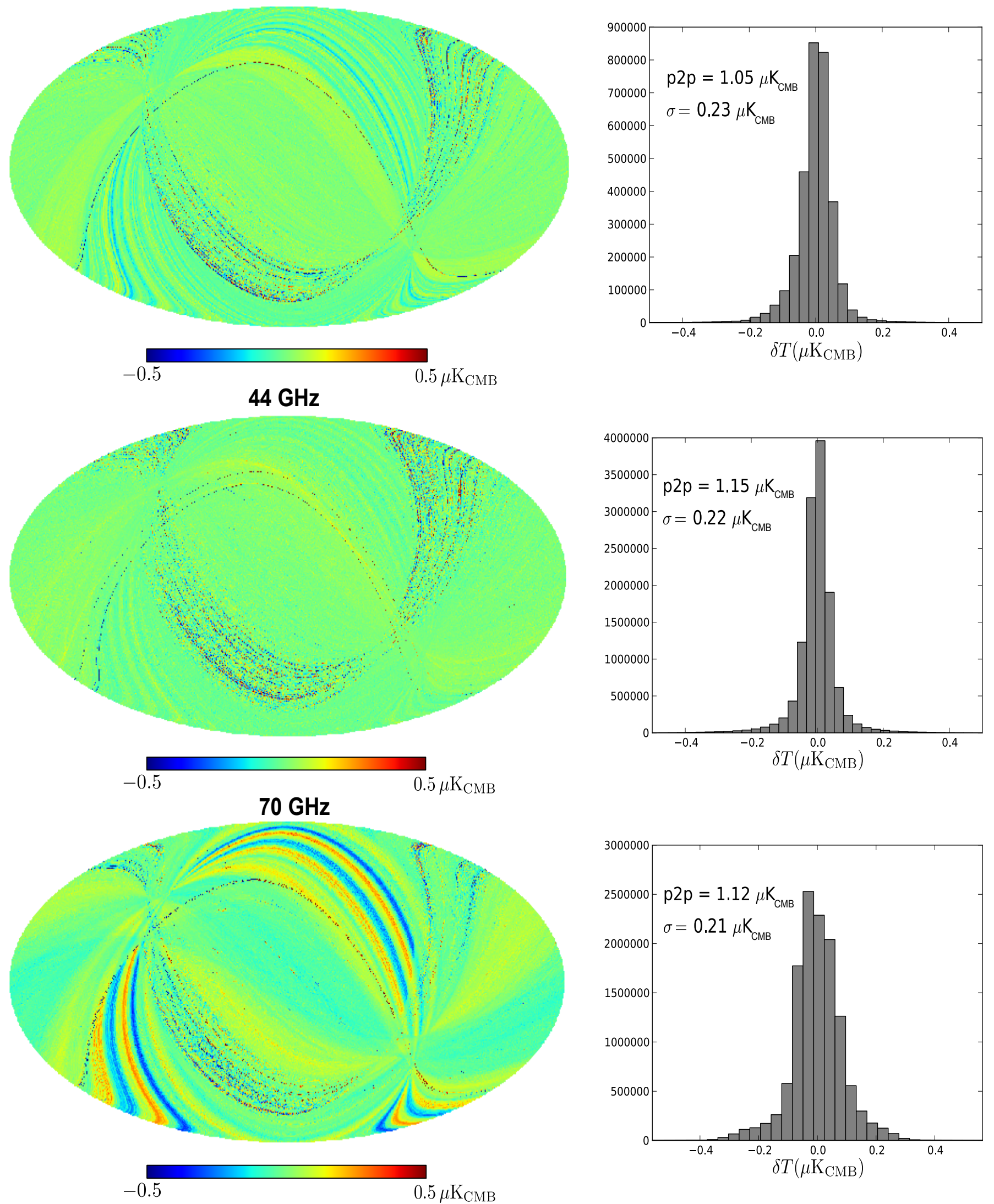

Fig. 30. Simulation of the systematic effects of temperature fluctuations of the LFI focal plane unit. Maps are $N_{\text {side }}=512$ at $30 \mathrm{GHz}$, and $N_{\text {side }}=$ 1024 at 44 and $70 \mathrm{GHz}$. 
A. Mennella et al.: Planck early results. III.

\section{$30 \mathrm{GHz}$}
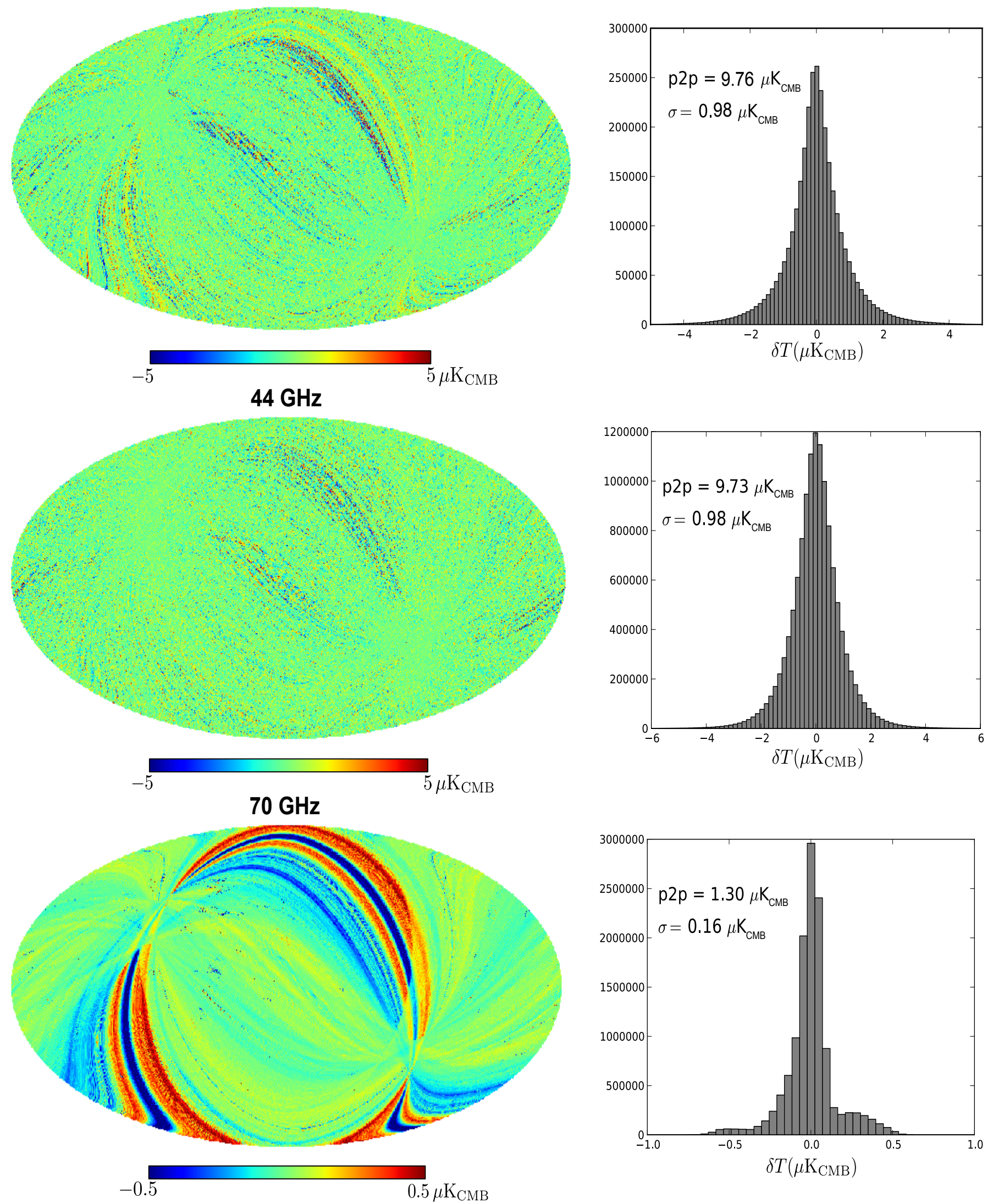

Fig. 31. Simulation of the systematic effects of temperature fluctuations of the $4 \mathrm{~K}$ reference loads. Maps are $N_{\text {side }}=512$ at $30 \mathrm{GHz}$, and $N_{\text {side }}=$ 1024 at 44 and $70 \mathrm{GHz}$. Note that the scale of 30 and $44 \mathrm{GHz}$ maps is 10 times larger than then scale of the map at $70 \mathrm{GHz}$. 


\section{References}

Bersanelli, M., Mandolesi, N., Butler, R. C., et al. 2010, A\&A, 520, A4 Cuttaia, F., Mennella, A., Stringhetti, L., et al. 2009, J. Instrum., 4, 2013 D’Arcangelo, O., Figini, L., Simonetto, A., et al. 2009, J. Instrum., 4, 2007 Davis, R. J., Wilkinson, A., Davies, R. D., et al. 2009, J. Instrum., 4, 2002 de Gasperis, G., Balbi, A., Cabella, P., Natoli, P., \& Vittorio, N. 2005, A\&A, 436,1159

Górski, K. M., Hivon, E., Banday, A. J., et al. 2005, ApJ, 622, 759

Gregorio, A., Mennella, A., Cuttaia, F., et al. 2011, The in-flight calibration and verification of the Planck-LFI instrument, J. Instrum. submitted Herreros, J. M., Gómez, M. F., Rebolo, R., et al. 2009, J. Instrum., 4, 2008 Hinshaw, G., Weiland, J. L., Hill, R. S., et al. 2009, ApJS, 180, 225 Jarosik, N., Bennett, C. L., Dunkley, J., et al. 2011, ApJS, 192, 14 Keihänen, E., Kurki-Suonio, H., Poutanen, T., Maino, D., \& Burigana, C. 2004, A\&A, 428, 287

Keihänen, E., Kurki-Suonio, H., \& Poutanen, T. 2005, MNRAS, 360, 390

Keihänen, E., Keskitalo, R., Kurki-Suonio, H., Poutanen, T., \& Sirviö, A. 2010, A\&A, 510, A57

Kogut, A., Banday, A. J., Bennett, C. L., et al. 1996, ApJ, 470, 653

Kurki-Suonio, H., Keihänen, E., Keskitalo, R., et al. 2009, A\&A, 506, 1511

Lamarre, J., Puget, J., Ade, P. A. R., et al. 2010, A\&A, 520, A9

Ludwig, A. 1973, IEEE Transactions on Antennas and Propagation, 21, 116

Mandolesi, N., Bersanelli, M., Butler, R. C., et al. 2010, A\&A, 520, A3

Maris, M., Tomasi, M., Galeotta, S., et al. 2009, J. Instrum., 4, 2018

Meinhold, P., Leonardi, R., Aja, B., et al. 2009, J. Instrum., 4, 2009

Mennella, A., Bersanelli, M., Seiffert, M., et al. 2003, A\&A, 410, 1089

Mennella, A., Villa, F., Terenzi, L., et al. 2009, J. Instrum., 4, 2011

Mennella, A., Bersanelli, M., Butler, R. C., et al. 2010, A\&A, 520, A5

Mennella, A., Bersanelli, M., Butler, R. C., et al. 2011, A\&A, 536, A3

Morgante, G., Pearson, D., Melot, F., et al. 2009, J. Instrum., 4, 2016

Natoli, P., de Gasperis, G., Gheller, C., \& Vittorio, N. 2001, A\&A, 372, 346

Planck Collaboration 2011a, A\&A, 536, A1

Planck Collaboration 2011b, A\&A, 536, A2

Planck Collaboration 2011c, A\&A, 536, A7

Planck Collaboration 2011d, A\&A, 536, A8

Planck Collaboration 2011e, A\&A, 536, A9

Planck Collaboration 2011f, A\&A, 536, A10

Planck Collaboration 2011g, A\&A, 536, A11

Planck Collaboration 2011h, A\&A, 536, A12

Planck Collaboration 2011i, A\&A, 536, A13

Planck Collaboration 2011j, A\&A, 536, A14

Planck Collaboration 2011k, A\&A, 536, A15

Planck Collaboration 20111, A\&A, 536, A16

Planck Collaboration 2011m, A\&A, 536, A17

Planck Collaboration 2011n, A\&A, 536, A18

Planck Collaboration 2011o, A\&A, 536, A19

Planck Collaboration 2011p, A\&A, 536, A20

Planck Collaboration 2011q, A\&A, 536, A21

Planck Collaboration 2011r, A\&A, 536, A22

Planck Collaboration 2011s, A\&A, 536, A23

Planck Collaboration 2011t, A\&A, 536, A24

Planck Collaboration 2011u, A\&A, 536, A25

Planck Collaboration 2011v, The Explanatory Supplement to the Planck Early

Release Compact Source Catalogue (ESA)

Planck Collaboration 2011w, A\&A, 536, A26

Planck HFI Core Team 2011a, A\&A, 536, A4

Planck HFI Core Team 2011b, A\&A, 536, A6

Poutanen, T., Maino, D., Kurki-Suonio, H., Keihänen, E., \& Hivon, E. 2004, MNRAS, 353, 43

Prunet, S., Ade, P. A. R., Bock, J. J., et al. 2001 [arXiv: astro-ph/0101073]

Sandri, M., Villa, F., Bersanelli, M., et al. 2010, A\&A, 520, A7

Seiffert, M., Mennella, A., Burigana, C., et al. 2002, A\&A, 391, 1185

Terenzi, L., Salmon, M. J., Colin, A., et al. 2009, J. Instrum., 4, 2012

Tomasi, M., Mennella, A., Galeotta, S., et al. 2009, J. Instrum., 4, 2020

Valenziano, L., Cuttaia, F., De Rosa, A., et al. 2009, J. Instrum., 4, 2006

Villa, F., Terenzi, L., Sandri, M., et al. 2010, A\&A, 520, A6

Zacchei, A., Maino, D., Baccigalupi, C., et al. 2011, A\&A, 536, A5

Zonca, A., Franceschet, C., Battaglia, P., et al. 2009, J. Instrum., 4, 2010

1 Aalto University Metsähovi Radio Observatory, Metsähovintie 114, 02540 Kylmälä, Finland

2 Agenzia Spaziale Italiana Science Data Center, c/o ESRIN, via Galileo Galilei, Frascati, Italy
3 Agenzia Spaziale Italiana, viale Liegi 26, Roma, Italy

4 Astroparticule et Cosmologie, CNRS (UMR7164), Université Denis Diderot Paris 7, Bâtiment Condorcet, 10 rue A. Domon et Léonie Duquet, Paris, France

5 Australia Telescope National Facility, CSIRO, PO Box 76, Epping, NSW 1710, Australia

6 CNRS, IRAP, 9 Av. Colonel Roche, BP 44346, 31028 Toulouse Cedex 4, France

7 California Institute of Technology, Pasadena, California, USA

8 Centre of Mathematics for Applications, University of Oslo, Blindern, Oslo, Norway

9 DA-Design Oy, Keskuskatu 29, Jokioinen, Finland

10 DTU Space, National Space Institute, Juliane Mariesvej 30, Copenhagen, Denmark

11 Departamento de Física, Universidad de Oviedo, Avda. Calvo Sotelo s/n, Oviedo, Spain

12 Departamento de Ingeniería de Comunicaciones, Universidad de Cantabria, Plaza de la Ciencia, 39005 Santander, Spain

13 Departamento de Matemáticas, Estadística y Computación, Universidad de Cantabria, Avda. de los Castros s/n, Santander, Spain

14 Department of Physics \& Astronomy, University of British Columbia, 6224 Agricultural Road, Vancouver, British Columbia, Canada

15 Department of Physics and Astronomy, University of Southern California, Los Angeles, California, USA

16 Department of Physics, Gustaf Hällströmin katu 2a, University of Helsinki, Helsinki, Finland

17 Department of Physics, University of California, Berkeley, California, USA

18 Department of Physics, University of California, One Shields Avenue, Davis, California, USA

19 Department of Physics, University of California, Santa Barbara, California, USA

20 Dipartimento di Fisica G. Galilei, Università degli Studi di Padova, via Marzolo 8, 35131 Padova, Italy

21 Dipartimento di Fisica, Università La Sapienza, P. le A. Moro 2, Roma, Italy

22 Dipartimento di Fisica, Università degli Studi di Milano, via Celoria, 16, Milano, Italy e-mail: aniello.mennella@fisica.unimi .it

23 Dipartimento di Fisica, Università degli Studi di Trieste, via A. Valerio 2, Trieste, Italy

24 Dipartimento di Fisica, Università di Ferrara, via Saragat 1, 44122 Ferrara, Italy

25 Dipartimento di Fisica, Università di Roma Tor Vergata, via della Ricerca Scientifica, 1, Roma, Italy

26 Dipartimento di Matematica, Università di Roma Tor Vergata, via della Ricerca Scientifica, 1, Roma, Italy

27 Dpto. Astrofísica, Universidad de La Laguna (ULL), 38206 La Laguna, Tenerife, Spain

28 European Space Agency, ESAC, Planck Science Office, Camino bajo del Castillo, s/n, Urbanización Villafranca del Castillo, Villanueva de la Ca nada, Madrid, Spain

29 European Space Agency, ESOC, Robert-Bosch-Str. 5, Darmstadt, Germany

30 European Space Agency, ESTEC, Keplerlaan 1, 2201 AZ Noordwijk, The Netherlands

31 Haverford College Astronomy Department, 370 Lancaster Avenue, Haverford, Pennsylvania, USA

32 Helsinki Institute of Physics, Gustaf Hällströmin katu 2, University of Helsinki, Helsinki, Finland

33 INAF - Osservatorio Astrofisico di Arcetri, Largo Enrico Fermi 5, Firenze, Italy

34 INAF - Osservatorio Astrofisico di Catania, via S. Sofia 78, Catania, Italy

35 INAF - Osservatorio Astronomico di Padova, vicolo dell'Osservatorio 5, Padova, Italy 
A. Mennella et al.: Planck early results. III.

36 INAF - Osservatorio Astronomico di Roma, via di Frascati 33, Monte Porzio Catone, Italy

37 INAF - Osservatorio Astronomico di Trieste, via G.B. Tiepolo 11, Trieste, Italy

38 INAF/IASF Bologna, via Gobetti 101, Bologna, Italy

39 INAF/IASF Milano, via E. Bassini 15, Milano, Italy

40 ISDC Data Centre for Astrophysics, University of Geneva, Ch. d'Écogia 16, Versoix, Switzerland

41 Infrared Processing and Analysis Center, California Institute of Technology, Pasadena, CA 91125, USA

42 Institut d'Astrophysique Spatiale, CNRS (UMR 8617) Université Paris-Sud 11, Bâtiment 121, Orsay, France

43 Institut d'Astrophysique de Paris, CNRS UMR 7095, Université Pierre \& Marie Curie, 98bis boulevard Arago, Paris, France

44 Institute of Astronomy, University of Cambridge, Madingley Road, Cambridge CB3 0HA, UK

45 Institute of Theoretical Astrophysics, University of Oslo, Blindern, Oslo, Norway

46 Instituto de Astrofísica de Canarias, C/vía Láctea s/n, La Laguna, Tenerife, Spain

47 Instituto de Física de Cantabria (CSIC-Universidad de Cantabria), Avda. de los Castros s/n, Santander, Spain

48 Istituto di Fisica del Plasma, CNR-ENEA-EURATOM Association, via R. Cozzi 53, Milano, Italy

49 Jet Propulsion Laboratory, California Institute of Technology, 4800 Oak Grove Drive, Pasadena, California, USA
50 Jodrell Bank Centre for Astrophysics, Alan Turing Building, School of Physics and Astronomy, The University of Manchester, Oxford Road, Manchester, M13 9PL, UK

51 LERMA, CNRS, Observatoire de Paris, 61 avenue de l'Observatoire, Paris, France

52 Lawrence Berkeley National Laboratory, Berkeley, California, USA

53 Max-Planck-Institut für Astrophysik, Karl-Schwarzschild-Str. 1, 85741 Garching, Germany

54 MilliLab, VTT Technical Research Centre of Finland, Tietotie 3, Espoo, Finland

55 National Radio Astronomy Observatory, 520 Edgemont Road, Charlottesville VA 22903-2475, USA

56 Nokia Corporation, Itämerenkatu 11-13, Helsinki, Finland

57 SISSA, Astrophysics Sector, via Bonomea 265, 34136, Trieste, Italy

${ }^{58}$ School of Electrical and Electronic Engineering, The University of Manchester, Sackville Street Building, Manchester, M13 9PL, UK

59 Space Sciences Laboratory, University of California, Berkeley, California, USA

60 Spitzer Science Center, 1200 E. California Blvd., Pasadena, California, USA

61 Thales Alenia Space Italia S.p.A., S.S. Padana Superiore 290, 20090 Vimodrone (MI), Italy

62 Université de Toulouse, UPS-OMP, IRAP, 31028 Toulouse Cedex 4, France

63 Warsaw University Observatory, Aleje Ujazdowskie 4, 00-478 Warszawa, Poland 